\title{
Data reduction and calibration accuracy of the imaging Fourier transform spectrometer SITELLE
}

\author{
T. Martin ${ }^{\oplus, 1,2 \star}$ L. Drissen ${ }^{1,2}$ and S. Prunet ${ }^{3,4}$ \\ ${ }^{1}$ Département de physique, de génie physique et d'optique, Université Laval, 2325, rue de l'université, Québec (Québec), G1V OA6, Canada \\ ${ }^{2}$ Centre de Recherche en Astrophysique du Québec (CRAQ), Montréal (Québec), H3C 3J7, Canada \\ ${ }^{3}$ Université Côte d'Azur, Observatoire de la Côte d'Azur, CNRS, Laboratoire Lagrange, Bd de l'Observatoire, CS 34229, F-06304 Nice cedex 4, France \\ ${ }^{4}$ Canada-France-Hawaii Telescope, 65-1238 Mamalahoa Hwy, Kamuela, HI 96743, USA
}

Accepted 2021 June 3. Received 2021 June 3; in original form 2021 March 19

\begin{abstract}
SITELLE, an imaging Fourier Transform Spectrometer, is part of the Canada-France-Hawaii instrument suite. It delivers spectral cubes covering an 11 arcmin $\times 11$ arcmin field of view with a seeing-limited spatial resolution and a tunable spectral resolution $(\mathrm{R} \sim 1-10000)$ in selected bands of the visible range (350-900 nm). We present a complete picture of the calibration accuracy obtained with the SITELLE processing pipeline ORBS. We put a particular emphasis on the description of our phase correction method and on the assessment of the flux calibration precision. We show that the absolute flux calibration uncertainty is to be considered between -15 percent and 5 percent. Flexure in the instrument is likely responsible for a wavelength calibration error gradient across the field of view, with an amplitude corresponding to 15 to $25 \mathrm{~km} \mathrm{~s}^{-1}$; measurements of the night-sky emission lines when present in a science cube reduces this error to $\sim 2 \mathrm{~km} \mathrm{~s}^{-1}$. The astrometric calibration is limited to $\sim 1$ arcsec by the optical distortions. Considering that imaging Fourier transform spectrometers are not as widely used as dispersive spectrometers and because SITELLE and its prototype are the first instruments of their kind to provide data in the near-UV at a high spectral resolution and over a very large field of view, we took great care in explaining most of the core concepts behind this technique as well as exploring all the practical limitations that affect the precision of our calibrations. As such, this paper aims at providing a solid ground for subsequent developments of imaging Fourier transform spectrometers in astronomy.
\end{abstract}

Key words: instrumentation: interferometers - methods: data analysis - techniques: imaging spectroscopy.

\section{INTRODUCTION}

SITELLE (Drissen et al. 2019), an imaging Fourier Transform Spectrometer, is part of the Canada-France-Hawaii instrument suite. It delivers spectral cubes covering an $11 \operatorname{arcmin} \times 11$ arcmin field of view with a seeing-limited spatial resolution and a tunable spectral resolution ( $\mathrm{R} \sim 1-10000)$ in selected bands of the visible range (350$900 \mathrm{~nm}$; see Fig. 1). SITELLE is based on an off-axis interferometer, which gives the opportunity to measure the flux on both output ports instead of one in the classical, on-axis, configuration (see Fig. 2; Grandmont et al. 2012).

Two $2 \mathrm{k} \times 2 \mathrm{k}$ CCD detectors are attached to the output ports. During an observing scan, the scanning mirror is moved. The optical path difference (OPD) between the two interfering beams is thus gradually changed, step by step, modulating the output intensity according to the spectral energy distribution of the observed source. The images collected at the output are interferometric images, which are stacked into two interferometric cubes (one for each output port). For a given pixel in the image, the intensity recorded during a scan of the moving mirror is an interferogram, the Fourier transform of which must be computed to determine the spectrum of the source.
More details on the observing process are given in Martin, Prunet \& Drissen (2016), Drissen et al. (2010), and references therein.

These two inteferometric cubes are transformed into one spectral cube by the processing pipeline ORBS. ${ }^{1}$ General concepts behind the pipeline process and the underlying software architecture can be found in Martin, Drissen \& Joncas (2012), Martin, Drissen \& Joncas (2015), and Martin (2015), but numerous algorithms and architectural improvements have since been implemented. The details of the operations of the pipeline that have an impact on the calibrations will be discussed in this article.

Ideally, the Fourier transform of all the recorded interferograms (around four million) would be the only operation required to transform an interferometric cube into a spectral cube of the same dimension. In practice, however, many operations on the raw data are actually needed to prepare and merge the interferograms obtained with each camera before being able to compute their spectrum and calibrate the obtained spectral cube.

The reduction pipeline delivers data with a basic reduction and calibration process. More accurate calibrations can be obtained by the observer for some data sets with the data analysis software ORCS ${ }^{2}$ (Martin et al. 2015, 2016; Shara et al. 2016; Martin, Drissen \& 


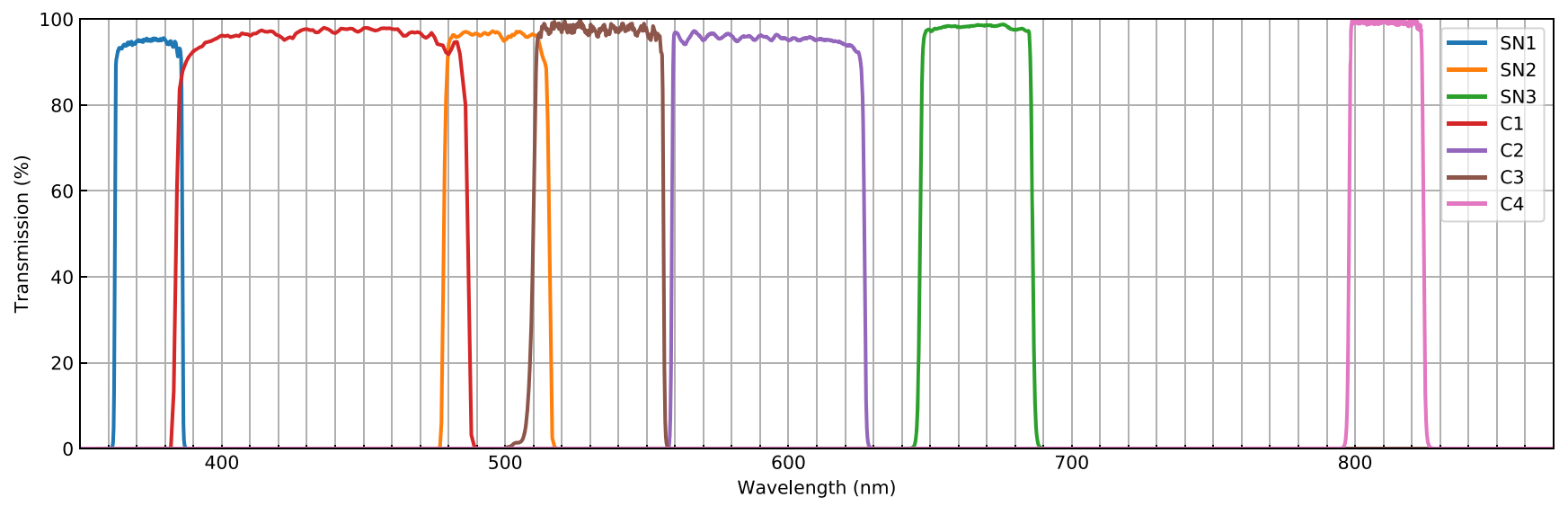

Figure 1. SITELLE's filters transmission curves provided by the manufacturer (measured at the centre of the filter with a $1 \mathrm{~nm}$ slit width). Due to the variation of the incident angle and the displacement of the beam on the surface of the filter itself (since the filter is not on the pupil plane), we observe a slight shift of the transmission curves $(<2 \mathrm{~nm})$ from the centre to the edge of the field of view. There is no need to compensate for this effect during the data reduction process, but it must be taken into account in subsequent analyses. The out of band rejection is smaller than 0.01 per cent from 300 to $1150 \mathrm{~nm}$.

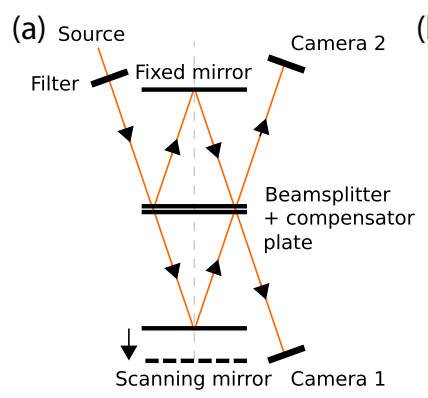

(b)

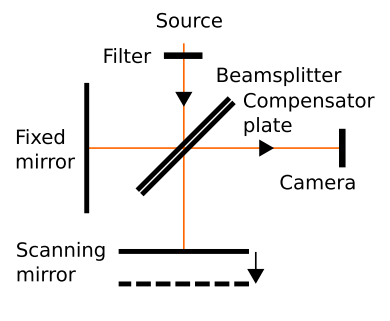

Figure 2. (a) Sketch of SITELLE's interferometer. (b) Sketch of a classical Michelson interferometer.

Melchior 2018; Rousseau-Nepton et al. 2017; Gendron-Marsolais et al. 2018), but since these calibrations depend on the objects observed and cannot be blindly applied to all the data cubes, they are not handled nor supported by the CFHT. The main focus of this article is to provide an accurate picture of SITELLE's data calibration quality as provided by the $C F H T$. Improvements on the calibrations will be introduced in future versions of the reduction pipeline. Section 2 provides an overview of the reduction pipeline. In Sections 3 and 4 we describe in detail the Fourier transform and the phase correction process, which can have a major impact on the flux and the wavelength calibrations. Flux, wavelength, and astrometric calibration are considered in Sections 5, 6, and 7, respectively.

Note that some of the most important results of this paper have already been presented in a conference proceedings (Martin \& Drissen 2016) but in a much less detailed fashion.

This article can also be considered as an introduction to our previously published articles about SITELLE data reduction and analysis (Martin et al. 2016, 2018). The basic formalism as well as core concepts are introduced and developed with some detail in the following section.

\section{REDUCTION PROCESS}

Each step of the reduction has an impact on the quality of the calibration, and especially the flux calibration. There have been some changes since the description of the pipeline presented in Martin et al. (2012, 2015) and Martin (2015). The current version of the data

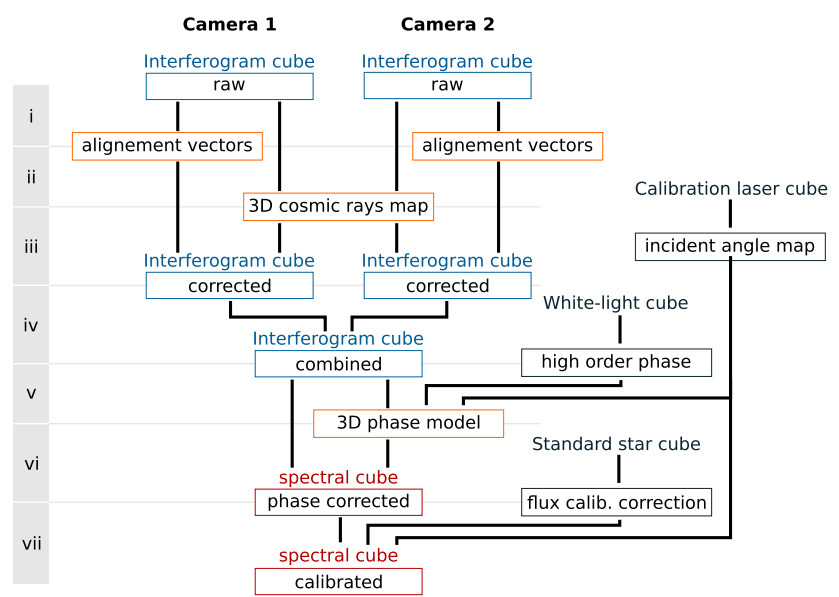

Figure 3. Processing pipeline workflow. The input and output of each step described in Section 2 are represented. Interferogram cubes are in blue, spectral cubes in red, internally computed data in orange, and data obtained from an external source in grey.

processing steps is as follows (see Fig. 3 for a graphical representation of the whole process):

(i) Computation of the alignment vectors to compensate for guiding errors during the scan (see Fig. 4). When possible, 15 stars are detected and the position of their centroid is measured in each image to estimate the horizontal and vertical shifts with respect to the first image. Centroids are measured via individual fit of a 2D Gaussian model on the detected stars. The precision on the computed shift is always better than 1/10th of a pixel. The alignment vectors obtained are used at step (iii). In general, the guiding errors are kept below 4 pixels, which is of the order of the maximum allowed displacement caused by mechanical flexure. ${ }^{3}$ A simulation of the observation of

${ }^{3}$ It was noticed at the beginning of SITELLE operations that the differential flexure between the instrumental field of view and the Cassegrain guide camera could exceed the requirements to stay below 1 arcsec. Nowadays, an astrometric solution is derived on each image of the interferogram sequence immediately after their acquisition, and if the observed relative astrometric 

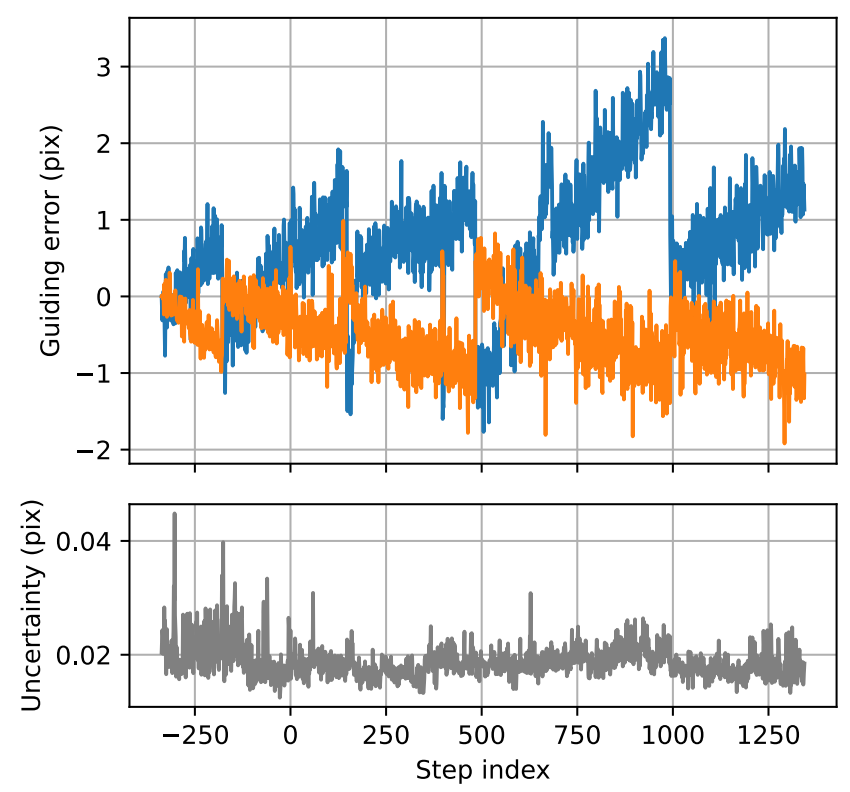

Figure 4. Top: Typical guiding error along the $x$ - (blue) and $y$ - (orange) axis of camera 1 . This is obtained by following the displacement of the centroid of 15 stars randomly chosen in the field of view. strong variations of the guiding error are due to compensation of the drift during the observation. Bottom: Uncertainty on the estimation of the guiding error.

a laser shows that it may be responsible for a loss of modulation efficiency of 2 per cent to 3 per cent (see equation 10).

(ii) Cosmic-rays detection. A 3D map of the detected cosmicrays is created for each cube. The detection process is described in Martin et al. (2015). The impact rate per image is around $5 \mathrm{~s}^{-1}$, i.e. $0.5 \mathrm{~s}^{-1} \mathrm{~cm}^{-2}$. Depending on the total exposure time, the number of affected spectra may thus represent up to a few percent of the field of view. False detections may appear in stellar interferograms since they are point-like and subject to abrupt changes from one step to the other, especially near the zero path difference (ZPD) where the modulation is the highest (see Fig. 5). This can be mitigated by removing detections around bright stars, but a few bright pointlike regions may still be affected. A false detection will generate a correction which, in star interferograms, is very difficult to do properly: the median of the neighbouring pixels, as used in the extended regions, is not a reliable estimation. The computed cosmic rays maps are used at step (iii).

(iii) Correction of the CCD images: detrending, alignment, cosmic-rays correction. Bias is computed from the overscan columns and subtracted, flat-field illumination difference are corrected. No dark frame is subtracted because the dark current is very low $\left(<2 \mathrm{e}^{-} \mathrm{h}^{-1}\right)^{4}$ and in this case dark frame subtraction would enhance the noise in the images without having a significant impact on the quality of the flux calibration (see Section 5.2.1). Images are realigned with the alignment vectors and cosmic-rays are corrected (see Martin et al. 2015 for more details on the cosmic-rays correction process). Note that seeing variations are not considered in the data reduction. We could convolve all images to the worst seeing measured

drift becomes larger than 1 arcsec a compensating term is sent to the guiding loop. This explains the seesaw form of the guiding error in Fig. 4.

${ }^{4}$ See the specifications of the e2v's back-illuminated $2 \mathrm{k} \times 2 \mathrm{k}$ scientific $\mathrm{CCD}$ sensor CCD230-42 at http://www.e2v.com/resources/account/download-dat asheet $/ 3828$.

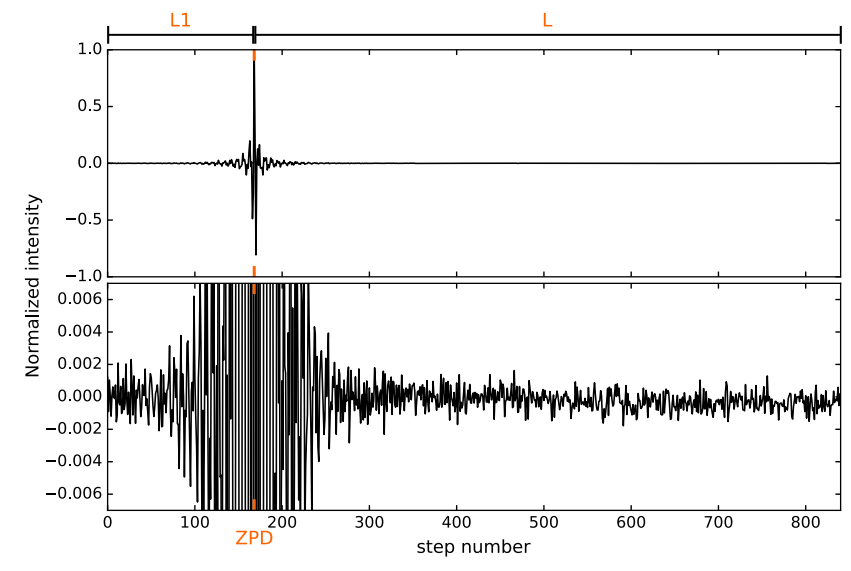

Figure 5. Example of a typical continuum source interferogram, obtained after step (iv) of the reduction process, and just before its transformation to a spectrum (see Section 2 for details). It was observed near the centre of M31 in the SN3 filter. Because SITELLE is a step-scan interferometer (i.e. the mirror moves to a target OPD, stops during the exposure, and moves to the next OPD), the horizontal axis gives the position of the moving mirror in terms of step number. The step size is $2943 \mathrm{~nm}$ in this case (in terms of OPD measured on the optical axis of the interferometer). The top panel shows the full interferogram and the bottom panel shows a zoomed-in version. The ZPD (the position where the OPD equals 0, here at step 168, indicated with an orange tick) is positioned at the highest measured intensity of the interferogram. The maximum OPD attained on the left side and the right side are, respectively, labeled $L_{1}$ and $L$ (see equations 8 and 9).

during a scan, but this problem only affects point-like sources (like stars or high-redshift galaxies) and is negligible below a resolution of 10000 (Mandar 2012), which is the highest resolution attained to date (Martin, Milisavljevic \& Drissen 2021).

(iv) Combination of the cubes. The two corrected interferometric cubes must be aligned and combined. The alignment is necessary since there is a slight optical misalignment between the cameras: a rotation of up to a few degrees $\left(\leq 3^{\circ}\right)$ plus a translation (under 1 percent of the field of view). As this misalignment is also expected to change when the cameras are removed and replaced for maintenance purpose the alignment parameters must be computed for each observation. Note that there is no particular mechanism for achieving arcsec precision on the optical rotation alignment of the cameras. The stars detected in the field of view are used to this end. Cubes are then combined to enhance the signal-to-noise ratio (SNR), correct for transmission variations of the sky during the observation, and remove the unmodulated background light (see Section 5.2.4).

(v) Estimation of the parameters of a 3D phase model (see Section 3).

(vi) Transformation of the combined cube to a spectral cube. The transformation is based on the Fast Fourier Transform (FFT) of each interferogram. The phase information obtained from the $3 \mathrm{D}$ phase model is used at this point (see Section 4).

(vii) Calibration of the spectral cube (see Sections 5, 6, and 7).

The impact of these reduction steps on the final calibration will be discussed throughout the paper.

\section{PHASE COMPUTATION}

Phase correction has an impact on both flux and wavelength calibrations (see e.g. Bell 1972). An incorrect phase correction will result in a distortion of the instrumental line shape (ILS) that will eventually lead to flux and wavelength errors (see the top of Fig. A1). 
For reference and for the sake of notation consistency between our different articles written about SITELLE data, we will start with some known formulae related to Fourier transforms.

If the source has a spectral distribution $S(\sigma)$, the intensity measured at an OPD $x$ will be (Sakai, Vanasse \& Forman 1968)

$I(x)=\int_{-\infty}^{+\infty} S(\sigma) e^{i \phi(\sigma)} e^{2 i \pi \sigma x} \mathrm{~d} \sigma$,

where $\phi(\sigma)$ is a phase error that may arise from dispersive effects in the optics of the interferometer and a non-perfect sampling. When both the fixed and the moving mirrors are at the same distance from the semi-reflective coating of the beamsplitter, the OPD $x$ is equal to 0 (this position is called the ZPD); in the absence of phase error $(\phi(\sigma)=0)$, the measured intensity is equal to the total intensity of the source. This means that, at ZPD, an interferogram is at its maximum intensity (see Fig. 5).

The Fourier transform of a non-corrected interferogram is therefore

$\hat{I}(\sigma)=S(\sigma) e^{i \phi(\sigma)}=\int_{-\infty}^{+\infty} I(x) e^{-2 i \pi \sigma x} \mathrm{~d} x$.

In the absence of phase errors, the spectrum corresponds to the real part of the Fourier transform. However, any uncorrected phase error will translate into an error in the calculated spectrum (see Fig. A1). A first approach consists in computing the power spectrum to remove the phase error

$P(\sigma)=|\hat{I}(\sigma)|^{2}=S(\sigma)^{2} e^{i \phi(\sigma)} e^{-i \phi(\sigma)}=S(\sigma)^{2}$.

However, the spectral resolution is thus reduced and the noise properties of the spectrum are much different as its distribution is squared. A better approach to recover the real spectrum is to compute the phase and remove it from the Fourier transform

$S(\sigma)=\operatorname{Re}\left(\hat{I}(\sigma) e^{-i \phi(\sigma)}\right)$.

The whole challenge of phase correction is therefore to recover the phase $\phi(\sigma)$, which is different from one interferogram to another, with the highest possible precision so that the source spectrum is perfectly recovered (i.e. its quality is only limited by photon and instrumental noises; p. 101, Davis, Abrams \& Brault 2001).

As the Fourier transform of an interferogram is complex, we can rewrite equation (4) as the sum of a real part and an imaginary part,

$\hat{I}(\sigma)=\hat{I}_{\operatorname{Re}}(\sigma)+i \hat{I}_{\operatorname{Im}}(\sigma)$.

The phase $\phi(\sigma)$ of the complex spectrum is, by definition,

$\phi(\sigma)=\tan ^{-1}\left(\frac{\hat{I}_{\mathrm{Im}}(\sigma)}{\hat{I}_{\mathrm{Re}}(\sigma)}\right)$.

If $\phi(\sigma)$ is not 0 , the corrected spectrum is calculated by removing the phase before the real part is returned (see equation 4).

In the absence of prior information, the phase is an arbitrary function of the wavenumber and can be different from one pixel to another (i.e. different for each interferogram of the cube). However, the common approach is to consider it as a slowly varying function of the wavenumber which, when computed from equation (6), contains some noise that must be removed before it is used to correct the spectrum (see e.g. Brault 1987). Optical dispersion and sampling errors yield a phase error that can in general be modelled using low order polynomials (Sakai et al. 1968),

$\phi(\sigma) \equiv p_{0}+p_{1} \sigma+p_{2} \sigma^{2}+O\left(\sigma^{3}\right)$.

If the interferogram is asymmetric, only the symmetric part around the ZPD is used to compute the phase. Ideally, one would try to obtain a symmetric interferogram to maximize the quality of the calculated phase since a symmetric interferogram introduces a separate ILS for the real and imaginary parts of the spectrum, but does not couple them as it leaves the sine and cosine modes orthogonal. However, as the spectral resolution depends on the maximum path difference attained with respect to the ZPD (see equation 9), the samples recorded symmetrically are only contributing to the SNR. Therefore, in order to maximize the attained resolution for a given total exposure time, SITELLE interferograms are not recorded symmetrically (for the same reasons, the Herschel/SPIRE FTS interferograms are also recorded asymmetrically; Fulton et al. 2016). The left side of the interferogram $\left(L_{1}\right)$ extends to 25 percent of the longest side $L$ (see Fig. 5). As the phase is computed with the samples that are distributed symmetrically around the ZPD (e.g. Bell 1972), the spectral resolution of the phase vector, $R_{\phi}$, depends on $L_{1}{ }^{5}$ (see e.g. Martin et al. 2016 for more details on the calculation of the resolution)

$R_{\phi}=\frac{2 \sigma L_{1}}{1.20671}$

while the resolution of the spectrum itself is four times higher,

$R=\frac{2 \sigma L}{1.20671}$

Ideally, a 3D phase cube could be directly obtained from the science target and used to compute the spectral cube, spectrum by spectrum, with no further considerations. However, the SNR of the phase vector is rarely high enough for such a straight approach to be used. Indeed, from equation (6), we can see that the precision of the phase measured at a given wavenumber depends on the SNR of the real part and the imaginary part of the spectrum measured at this wavenumber. As the noise is equally distributed over all the channels, the precision of the phase calculated at a given wavenumber is directly proportional to the flux of the source at this same wavenumber. An emission-line spectrum, typical of an $\mathrm{H}$ II region, will therefore produce a reliable phase information only at the wavelengths of the lines, while a continuum source (like a star, a galaxy, or the earth atmosphere) will be much better at providing a reliable phase in the whole observed spectral band (Learner et al. 1995).

Consequently, the quality of the measured phase value will be different from one channel to another and also from one spectrum to another depending on the observed object. In some cases a reliable phase can be obtained everywhere in the field of view (e.g. when targeting high-redshift galaxies, elliptical galaxies, etc.), but targets for which most of the signal is concentrated into a few emission lines will produce unreliable phase data at least in a sub-region of the field of view. As a consequence, at least for these targets, the phase must be computed from an external continuum source. We have thus decided to compute a 3D phase model calibrated with a scan of a white-light source to correct the phase of all the obtained science cubes. The number of steps is set to 1000 on the longest side of the interferogram (i.e. a total of 2001 steps since the interferograms must

\footnotetext{
${ }^{5}$ There is a side effect when computing the phase from a low resolution spectrum: equation (6) is an unbiased estimator of the phase only in the limit of infinite resolution, otherwise it gets biased by the respective ILS of the real and imaginary parts of the observed spectrum when computing their ratio. This effect is negligible on continuum spectra, but gives serious artefacts on the estimation of the phase on emission lines. As mentioned below, when observing extended bright emission-lines regions, the phase estimation must be done on a separate calibration source.
} 

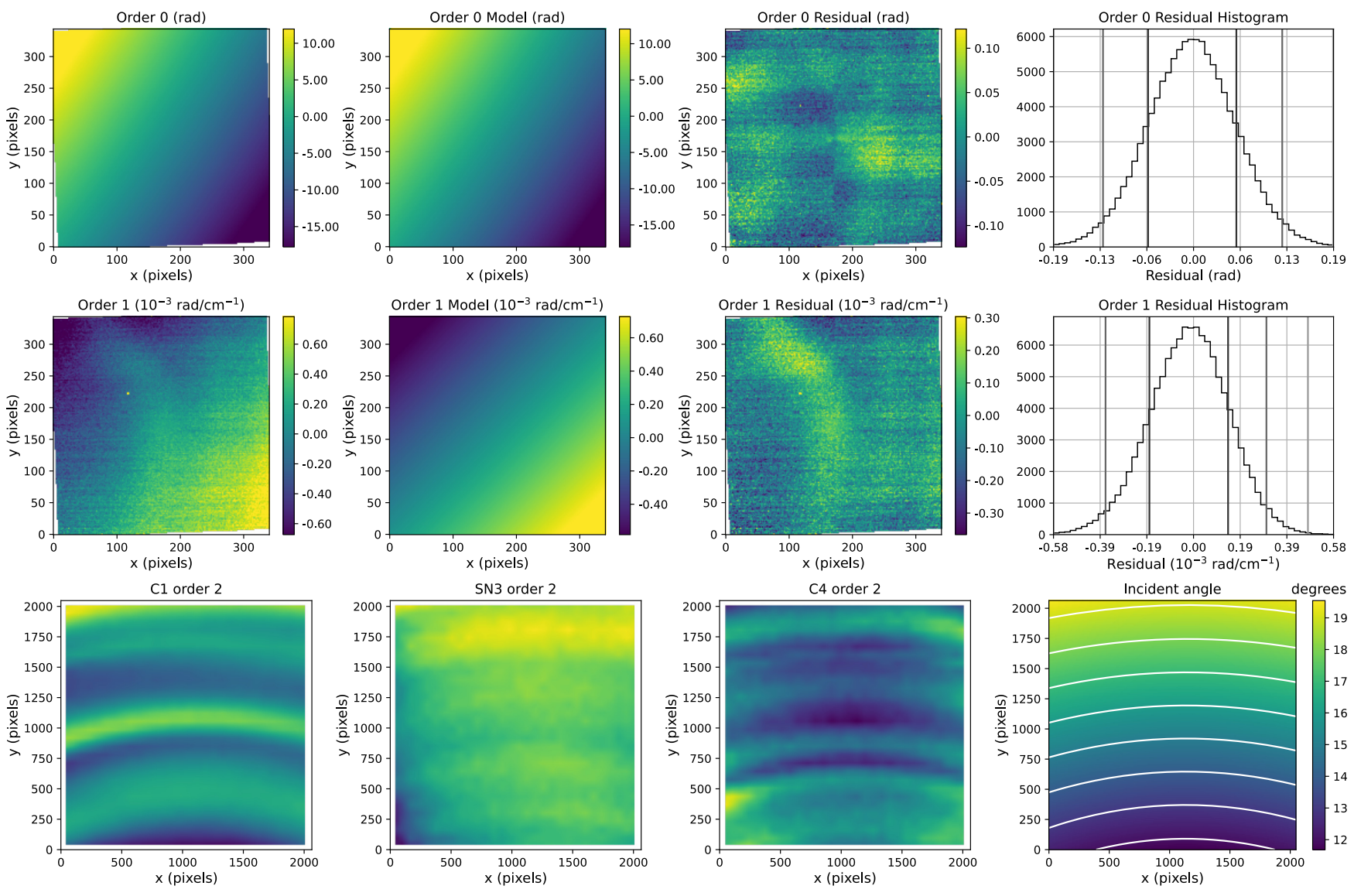

Figure 6. Modeling of the order 0 (top row) and 1 (central row) phase maps with a Zernike polynomial model. These phase maps are obtained from a linear fit of the residual phase cube once the high order phase cube is subtracted. The high order phase cube is obtained from the observation of a white light source. These maps were fitted on the science target M 95 obtained in the SN1 filter. The original map, the model, the residual, and its histogram are, respectively, shown from left to right. Note that pixels are binned $6 \times 6$ to enhance the SNR. We clearly see a small residual that changes from one scan to another. The effects of phase errors on the recovery of the emission line parameters are discussed in Appendix A. Bottom row: Examples of higher order (here the order 2) phase maps obtained from a white light source in various filters. These maps are used as is in the phase modeling. Only the first two orders are fitted to adapt the model to each science target. We can clearly see a dependency of the high order phase on the incident angle (bottom right-hand panel), but this is not sufficient to build a precise model on this single parameter.

be symmetric). The resolution thus vary from one filter to another (from 2000 in the $\mathrm{C} 1$ filter to 10000 in the $\mathrm{C} 4$ filter). As the phase is fitted with a low order polynomial, the resolution of the phase spectra can be much lower than the target resolution. A higher number of steps, and thus a higher resolution, is only important to maximize the SNR since CCDs will saturate above $\sim 65000$ counts, which limits the exposure time. ${ }^{6}$

For each filter, the phase of a white-light cube is thus obtained. This phase cube is fitted with polynomials to convert it as a set of maps $\left(N+1\right.$ maps if a polynomial of order $N$ is fitted). ${ }^{7}$ The order of the polynomial is between 3 and 5 except for the $\mathrm{C} 1$ filter for which an order 8 polynomial is necessary. The obtained 3D model has yet to be adapted to each science target since: (1) the position of the sampling vector may be shifted with respect to the real ZPD position, which is responsible for an added linear phase term and (2) the optics alignment changes slightly with the environmental conditions and the orientation of the gravity vector. We have found

${ }^{6}$ Note that below the saturation limit, CCDs are perfectly linear.

${ }^{7}$ Because the precision of the phase computed at any given wavenumber is proportional to the intensity of the source at that wavenumber, all phase fitting procedures are weighted by the intensity of the source. that these changes were only affecting the first two orders of the phase model.

Therefore, to adapt the calibrated phase model to the science target, we start by subtracting the calibrated phase from the target phase cube. We obtain a linear residual, which can be fitted with an order 1 polynomial everywhere in the field where the phase is reliable enough. The two phase maps obtained (the order 0 and order 1 maps) for the science target can be fitted with a Zernike polynomial model to estimate it where it could not be reliably computed. Since the camera orientation or the environmental conditions may change from one run to the other, phase calibration cubes are generated for each run. This ensures that a Zernike model can be used to compensate for flexures (due to the direction of the telescope) and small temperature drifts during a run.

The $3 \mathrm{D}$ phase model for each science target is thus a set of phase maps. The order 0 and order 1 maps, which were obtained from the science target cube itself and the orders greater than 1 , which come from a white-light cube. As an example we show in Fig. 6 the order 0 and order 1 maps obtained on the spiral galaxy M 95 in the SN1 filter $(R \simeq 1000$ ). We clearly see a small residual, which changes from one scan to another. This non-modeled bias is responsible for an error below 1 per cent in flux and a velocity shift below 10 per cent of the target resolution (e.g. $3 \mathrm{~km} \mathrm{~s}^{-1}$ at $R=10000$ ) in the worst case 
Table 1. Typical $3 \sigma$ phase error in different filters and its consequences on the measured flux and position of the emission lines. The shift error depends on the resolution and is thus given in percentage of the full width at half-maximum (FWHM). The $3 \sigma$ error is returned since the errors are not normally distributed (see Fig. 6). The effects of phase errors on the recovery of the emission line parameters are discussed in appendix A.

\begin{tabular}{lccc}
\hline Filter & $\begin{array}{c}3 \sigma \text { phase error } \\
(\mathrm{rad})\end{array}$ & $\begin{array}{c}\text { Flux error } \\
(\%)\end{array}$ & $\begin{array}{c}\text { Shift error } \\
(\% \text { FWHM })\end{array}$ \\
\hline SN1 & 0.19 & 0.47 & 7.6 \\
SN2 & 0.10 & 0.13 & 3.9 \\
SN3 & 0.12 & 0.18 & 4.7 \\
C1 & 0.24 & 0.72 & 9.4 \\
C2 & 0.20 & 0.48 & 7.6 \\
C3 & 0.090 & 0.10 & 3.5 \\
C4 & 0.039 & 0.019 & 1.5 \\
\hline
\end{tabular}

(C1 filter; see Table 1). The effects of a phase error on the measured flux and position of the emission line are discussed in appendix A. The worst results are obtained in the widest $(\mathrm{C} 1, \mathrm{C} 2)$ and bluest (SN1) filters. This is expected since in the blue, the variations of the refractive index with the wavelength in the beamsplitter are the most important and, at the same time, the quality of the optics (especially the surface flatness) becomes more and more significant. Examples of the high orders phase maps (orders greater than 1) obtained from a white light source are shown in the bottom row of Fig. 6. We can clearly see a dependency on the incident angle (see bottom right-hand panel of the same figure), which has been used in previous versions of the reduction code to build a model of the high order phase on this single parameter. However, we realized that it was not precise enough, especially in the corners. We thus decided to use the whole $3 \mathrm{D}$ high order phase cube and adapt it to the science targets by fitting only the two first orders, as explained above. The median of the 3D high order phase cube is shown in Fig. 7 for all SITELLE filters.

\section{FOURIER TRANSFORM}

To obtain a spectral cube (i.e. a cube of spectra) from an interferometric cube (i.e. a cube of interferograms), each one of the four million interferograms is individually Fourier transformed in an iterative fashion. A typical interferogram obtained after step (iii) of the reduction process (see Section 2) is shown in Fig. 5.

The transformation of an interferogram into its spectrum is the core of the reduction pipeline. All the preceding steps (images detrending, cubes alignment and combination, and phase computation) are mostly performed to enhance the quality of the interferograms before this operation.

Besides the known direct Fourier transform operation, a number of other operations on the interferogram are required to obtain a reliable spectrum (see e.g. Davis et al. 2001, ch. 6, and Fulton et al. 2016). These operations are:

(i) Subtraction of the mean of the whole interferogram to remove spurious signals in the first channel of the calculated spectrum.

(ii) Multiplication by a ramp-like truncation function. This operation was proposed by Mertz (1967) for the processing of asymmetric interferograms. The author shows that the non-observed samples on the left side should be replaced with zeros and the symmetric portion around the ZPD should be multiplied with a ramp-like function to avoid giving twice more weight to the left-side samples (see the top of Fig. 8). We decided not to use the mathematically equivalent Forman method (Forman, Steel \& Vanasse 1966) because, even if this method
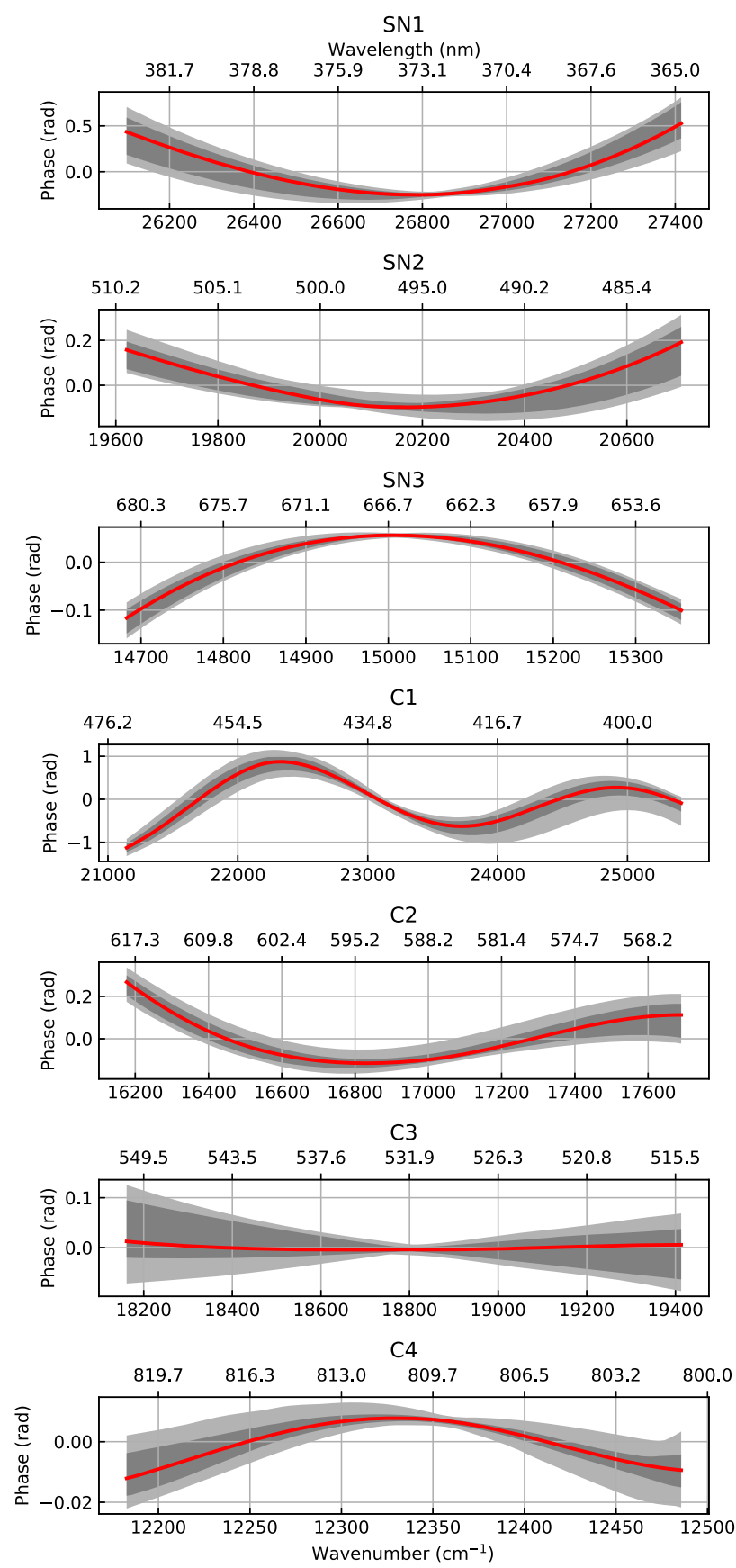

Figure 7. High order phase obtained from a white light source for all SITELLE filters. The red curve represents the median of the phase in the obtained high order phase cube. The regions in dark grey and light grey represents, respectively, the $1 \sigma$ and $3 \sigma$ limits.

results in a better spectral reconstruction on interferograms with more than a few thousand points (e.g. Sakai et al. 1968; Michaelian 1989), it implies the loss of at least a few hundred points on both sides of the interferogram (Chase 1982) to be effective. Since our interferograms are usually less than a thousand points long it would thus have a too great impact on the SNR and the attained resolution given the small enhancement on the reconstruction ( 0.2 percent, Michaelian 1989).

(iii) Zero padding. This is only required when using the FFT algorithm and avoids losing information during the transform. 

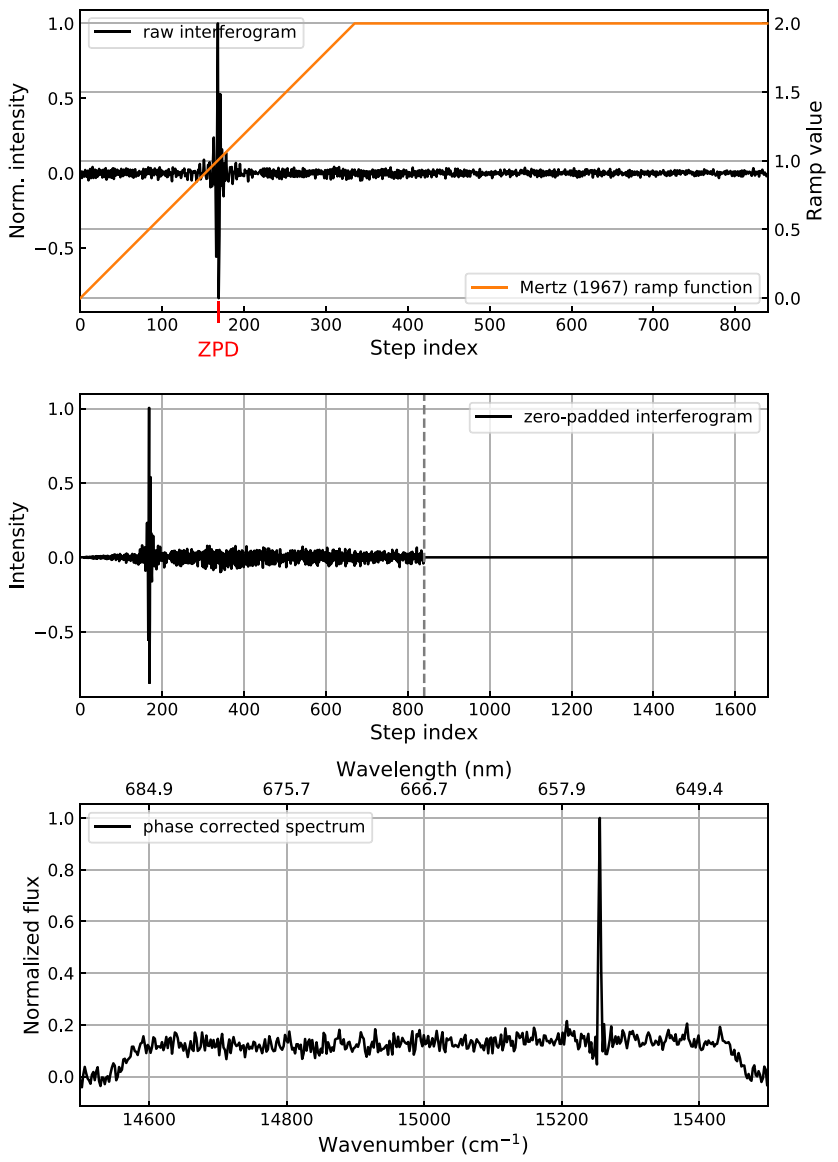

Figure 8. Illustration of the Fourier transform process on the interferogram of a planetary nebula observed in the SN3 cube of M 31. This interferogram is also shown in Fig. 5. It contains a good deal of continuum emission coming from the old stellar population. Top: The $N=840$ steps long raw interferogram and the ramp-like truncation function suggested by Mertz (1967). The position of the ZPD is indicated. Center: The zero-padded interferogram where $N$ zeroes have been concatenated to the right side. The resulting zero-padded interferogram is thus $2 N=1680$ steps long. The grey dotted line indicates the beginning of the padded region. Bottom: Real part of the phase corrected spectrum obtained after the Fourier transform. The borders of the SN3 filter can be seen below $14600 \mathrm{~cm}^{-1}$ and above $15450 \mathrm{~cm}^{-1}$. An $\mathrm{H} \alpha$ emission line having a radial velocity around $-350 \mathrm{~km} \mathrm{~s}^{-1}$ is clearly visible at 15255 $\mathrm{cm}^{-1}(655.5 \mathrm{~nm})$.

When taking the real part of the obtained spectrum, the interferogram must be filled with at least as much zeroes as the number of measured samples (see Davis et al. 2001, p. 99). In our case, if the original interferogram contains $\mathrm{N}$ samples, the zeropadded interferogram contains $2 \mathrm{~N}$ samples (see the middle panel of Fig. 8).

(iv) Fourier transform via an FFT algorithm (Cooley \& Tukey 1965) that outputs a complex spectrum. The complex spectrum obtained from the FFT algorithm ( $2 \mathrm{~N}$ channels) is split in two halves and only the left part ( $\mathrm{N}$ samples) is kept since, the interferogram being a real signal, the right part is a mirror of the left part across the Nyquist frequency (found at channel N). The obtained spectrum is $\mathrm{N}$-channel long and complex.

(v) Phase correction, which returns a real, phase-corrected spectrum. Phase correction is described in detail in Section 3. After the phase correction, only the real part is kept (see the bottom of Fig. 8).
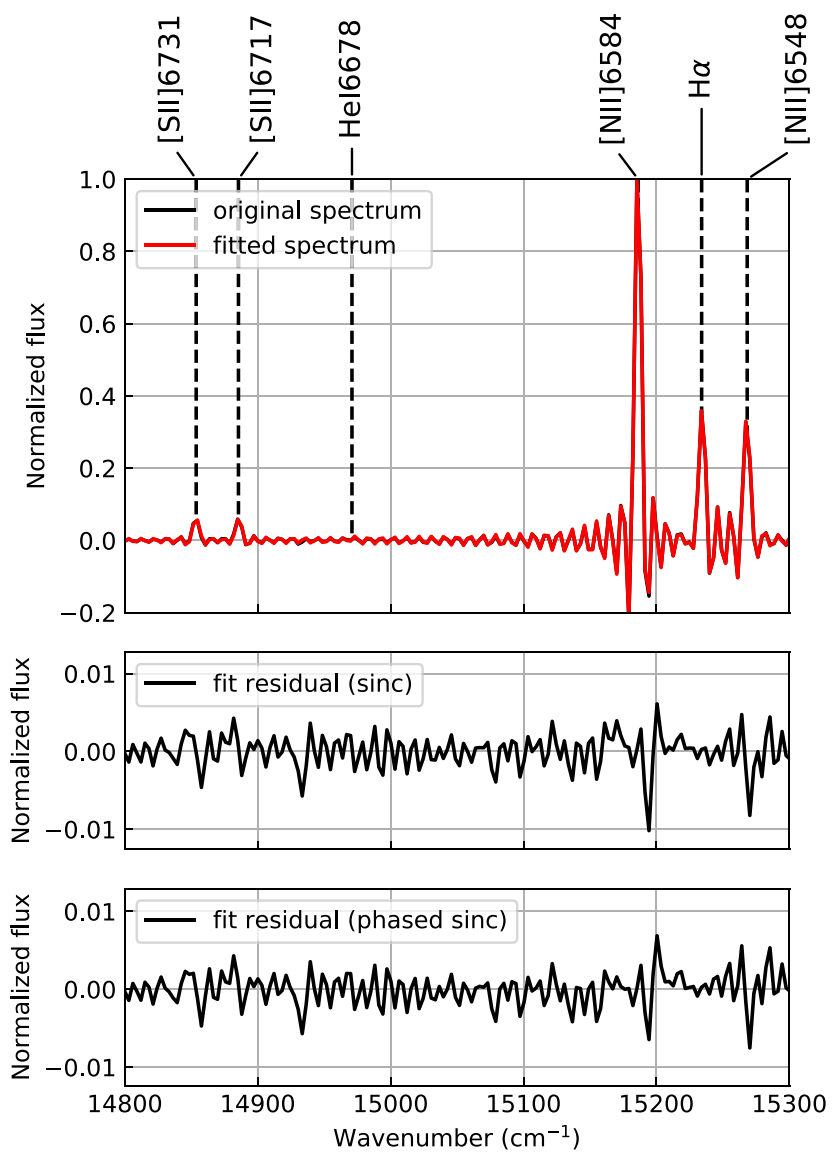

Figure 9. Example of a fit realized with ORCS (Martin et al. 2015) on a spectrum of a bright knot of the planetary nebula M57 (Martin et al. 2016) in the SN3 filter. Top: The fitted spectrum (in red) is superimposed on the original spectrum (in black). Centre: Residual from a fit with a pure sinc model. A 1 per cent modeling error is visible near the right lobe of the [N II] lines. Bottom: Residual from a fit with a phased sinc model. The modeling error is reduced but not eliminated suggesting that the residual cannot be explained only by a phase error.

Fig. 8 shows the results of the zero-padding, the multiplication by a ramp function, and the Fourier transform with phase correction.

\subsection{Instrumental line shape}

The ideal ILS of a phase corrected Fourier transform spectrum is a sinc function. Any error in the phase correction will result in a deformation of the ILS (see Fig. A1). Non-symmetric modulation efficiency loss with the OPD (see Section 4.2) can also generate an asymmetric ILS that will eventually be the source of wavelength and flux errors. The ILS is very well described by the theoretical model used to fit the emission lines described in Martin et al. (2016) despite a small modeling error that can be detected by fitting high SNR spectra. This error is located near the right lobe of the sinc and its amplitude, with respect to the central lobe, is around 1 percent (see Fig. 9). If this error was produced by a phase error, a negative residual on the right lobe of the sinc would be compensated by a positive residual on the left lobe, which is not the case. In the same figure, we show the result of a phased model (i.e. a pure sinc model multiplied by a constant phase term $\exp (i \phi))$; we can see that the residual error is reduced but not eliminated, suggesting that the ILS is not a perfect sinc. 
Table 2. Mean values of the terms in equation (12) for each SITELLE filter. Telescope transmission is based on the values provided by the CFHT. The atmospheric transmission at one airmass comes from Buton et al. (2012) The optics transmission have been provided by ABB. ME has been computed from the performance model of Mandar (2012) with jitters values adapted to fit the mean $\mathrm{ME}$ values provided by ABB. Note that there are some differences with the previous estimates reported in fig. 9 of Grandmont et al. (2012). The mean value of $\epsilon$ has been calculated from the data shown in Fig. 11. The mean inverse sensitivity $f_{\lambda}$ gives the flux density in erg cm ${ }^{-2} \mathrm{~s}^{-1} \AA^{-1}$ for which we measure an instrumental flux of one count per second per frequency channel.

\begin{tabular}{lccccccc}
\hline Filter & SN1 & SN2 & SN3 & C1 & C2 & C3 & C4 \\
\hline$t_{\text {atm. }}$ & 0.73 & 0.89 & 0.95 & 0.83 & 0.91 & 0.90 & 0.98 \\
$t_{\text {tel. }}$ & 0.86 & 0.85 & 0.81 & 0.86 & 0.83 & 0.84 & 0.75 \\
$t_{\text {opt. }}$ & 0.67 & 0.66 & 0.65 & 0.69 & 0.69 & 0.71 & 0.83 \\
$\mathrm{QE}$ & 0.69 & 0.88 & 0.92 & 0.90 & 0.90 & 0.88 & 0.86 \\
$\mathrm{ME}$ & 0.76 & 0.82 & 0.84 & 0.80 & 0.82 & 0.84 & 0.90 \\
$t$ & 0.21 & 0.34 & 0.37 & 0.34 & 0.36 & 0.38 & 0.46 \\
$\bar{\epsilon}$ & 0.59 & 0.95 & 1.05 & 0.93 & 1.00 & 1.06 & 1.00 \\
$\overline{f_{\lambda}} / 10^{-16}$ & 17.60 & 2.86 & 1.74 & 1.51 & 1.52 & 2.02 & 1.49 \\
\hline
\end{tabular}

\subsection{Modulation efficiency}

All the photons coming from the source will not interfere with the exact same phase. Differences in the optical flatness of the beamsplitter combined with the vibration of the mirrors during the exposure (jitter) produces small phase differences of the interfering photons that results in a loss of contrast of the fringes. This loss of contrast is quantified by the modulation efficiency (ME). It can be calculated from the maximum $I_{\max }$ and minimum $I_{\min }$ intensities attained during the modulation of a laser source of wavelength $\lambda$.

$\mathrm{ME}(\lambda)=\frac{I_{\max }-I_{\min }}{I_{\max }}$.

Because the quality of the optics and the jitter will not have the same impact at all wavelengths, the modulation efficiency depends on the wavenumber and is expected to decrease at smaller wavelengths. It can be modeled (Mandar 2012), but its precise measurement can only be obtained by observing a monochromatic source of light at the wavelength of interest (see fig. 4 of Drissen et al. 2019). The expected mean ME in all SITELLE filters is given in Table 2 .

The modulation efficiency can also suffer from pixel-to-pixel variations for various optical reasons and is known to decrease with the spectral resolution (Baril et al. 2016). We show in Fig. 10 two maps of the modulation efficiency obtained at $\mathrm{R}=5000$ and $\mathrm{R}=$ 10000 from the observation of a green $\mathrm{He}-\mathrm{Ne}$ laser at $\lambda=543.5$ $\mathrm{nm}$. We see that the ME decreases in the corners of the field of view and that this tendency is more pronounced at a higher resolution. Under $\mathrm{R}=5000$ a 3 percent gradient is observed (which is likely to be smaller at even lower resolution), but at 10000 this gradient is around 10 per cent.

\section{FLUX CALIBRATION}

\subsection{Flux calibration method}

Flux calibration is based on the measurement of the spectrum of a spectrophotometric standard star in each filter. The obtained spectrum is used to correct for the wavelength dependent transmission of the instrument and the telescope. A set of images of a standard star

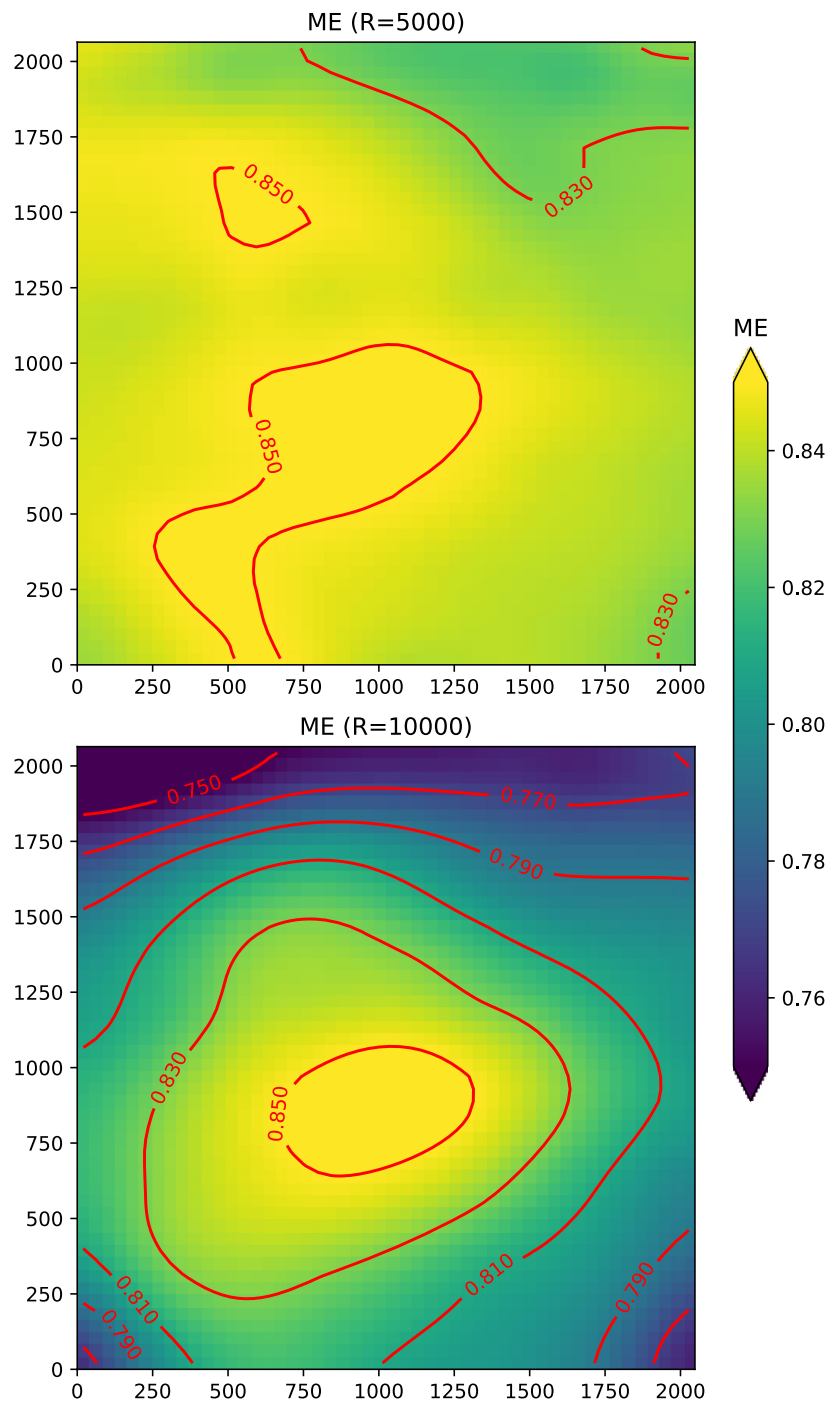

Figure 10. Mean modulation efficiency computed from the observation of a green He-Ne laser $(\lambda=543.5 \mathrm{~nm}$ ) at $\mathrm{R}=5000$ (top) and $\mathrm{R}=10000$ (bottom). The cube was recorded with a step size of $3255 \mathrm{~nm}$ and a folding order of 11 . The reasons why we observe a non-constant distribution of the modulation efficiency across the field of view that changes with the OPD are still not completely understood even if some effort have been made to assess this problem (Baril et al. 2016).

is also obtained for each scan. They are used to obtain an absolute flux calibration.

Most of the concepts considered in the following sections are not new. But, as SITELLE is at the same time an imager and a spectrometer, we must rely on hybrid methods for its flux calibration. We have thus chosen to provide detailed explanations as it will certainly help users to clearly understand the calibration process.

Flux calibration consists in computing the flux density $f_{\lambda}(\sigma)$ (in $\mathrm{erg} \mathrm{cm} \mathrm{cm}^{-2} \mathrm{~s}^{-1} \AA^{-1}$ ) for which we measure an instrumental flux $n(\sigma)$ in the calculated spectrum of 1 count per second at a given wavenumber. ${ }^{8}$ Following, for example, the same concepts as those

${ }^{8}$ The inconsistency of the unit of flux density used here, which is given per $\AA$ instead of being given per $\mathrm{cm}^{-1}$, which would better suit a spectrum calculated on an axis in wavenumber, is only apparent for the flux density is 
presented in the calibration of Hubble Space Telescope data, $f_{\lambda}(\sigma)$ is the inverse sensitivity. The whole idea is to compute $f_{\lambda}(\sigma)$ so that the flux density $f(\sigma)$ of an observed source can simply be related to $n(\sigma)$ via

$f(\sigma)=n(\sigma) f_{\lambda}(\sigma)$.

In general, we can estimate $n(\sigma)$ from $f(\sigma)$ with

$n(\sigma)=\frac{f(\sigma)}{\sigma h c} S_{\text {prim. }}(\sigma) t(\sigma) \epsilon(\sigma) \operatorname{ME}(\sigma) G$,

where $h$ is the Planck constant, $c$ the speed of light, $S_{\text {prim. }}$ the surface of the primary mirror, $G$ the CCD camera gain, $t$ the instrumental transmission, ME the modulation efficiency, and $\epsilon(\sigma)$ the flux correction function that should ideally be equal to unity if our knowledge of the instrument was perfect.

The instrumental transmission, which does not take the modulation efficiency into account, is the product of the transmission of all the optical components placed between the source and the detector,

$t(\sigma)=t_{\text {atm. }}(\sigma) t_{\text {tel. }}(\sigma) t_{\text {opt. }}(\sigma) t_{\text {filter }}(\sigma) \mathrm{QE}(\sigma)$,

where $t_{\mathrm{atm}}, t_{\mathrm{tel} .}, t_{\mathrm{opt}}$ and $t_{\text {filter }}$ are respectively the transmission coefficients of the atmosphere, the telescope optics, SITELLE's optics, and the filter. QE is the quantum efficiency of the CCD. Note that the airmass during the scan is taken into account in the atmospheric transmission term. Typical values of the different terms for each filter are given in Table 2.

Let $N_{\mathrm{i} \text {, obs }}$ be the instrumental flux of the source measured on the detectors (i.e. the number of photons collected on both cameras per second), we have

$N_{\mathrm{i}, \mathrm{obs}}=\int_{\sigma} \frac{f(\sigma)}{\sigma h c} S_{\text {prim. }}(\sigma) t(\sigma) \epsilon(\sigma) G$.

The instrumental flux in the calculated spectrum of the source $N_{\mathrm{s} \text {, obs }}$ will then be

$N_{\mathrm{s}, \mathrm{obs}}=\int_{\sigma} n(\sigma) \mathrm{d} \sigma=\overline{\mathrm{ME}} \times N_{\mathrm{i}, \mathrm{obs}}$,

where $\overline{\mathrm{ME}}$ is the mean modulation efficiency in the filter bandpass which acts, in this context, exactly as a transparency term.

From equations (11) and (12), we have

$f_{\lambda}(\sigma)=\frac{\sigma h c}{S_{\text {prim. }}(\sigma) t(\sigma) \epsilon(\sigma) \operatorname{ME}(\sigma) G}$,

With a classical spectrometer, $f_{\lambda}$ can be measured at will via the observation of a standard star. However this method cannot be used with SITELLE, since it takes a long time to collect a single standard star cube. We thus have to rely on the observation of the spectrum of a standard star in each filter and the acquisition of a set of images of a standard star for each scan.

With the standard spectrum, taken during a photometric night, we can estimate $\epsilon(\sigma)$ and thus $f_{\lambda}$. We then work under the hypothesis that, during the same run, the variations of $\epsilon$ from one scan to another are only due to the absorption of the atmosphere, which is assumed to be 'grey' (i.e. independent of the wavelength in the filter band-pass). In this case, measuring the mean value of $\epsilon(\sigma)$ is enough to correct $f_{\lambda}$ for the atmospheric transmission.

not, by definition, an integrated flux but a monochromatic flux. We have thus chosen to compute it per $\AA$ because this unit is widely used.

\subsubsection{Wavelength-dependent flux calibration}

The acquisition of the spectrum of a standard star under photometric conditions with SITELLE gives the possibility to compute the instrumental flux $n_{\mathrm{obs}}(\sigma)$. In addition, this spectrum can also be simulated via the knowledge of the different terms in equation (12) (considering $\epsilon(\sigma)=1$ ) to obtain $n_{\text {sim }}(\sigma)$. We may then calculate $\epsilon$ since

$\epsilon(\sigma)=\frac{n_{\mathrm{obs}}(\sigma)}{n_{\mathrm{sim}}(\sigma)}$,

which leads to an estimate of $f_{\lambda}$ under the same atmospheric conditions. Fig. 11 shows, for each filter, the observed spectrum of one standard star, its simulation, and the obtained estimation of $\epsilon$. From this results we can conclude that our knowledge of the instrumental transmission functions is very good for all the filters with the exception of SN1 and C1. These filters are in the near UV (360-390 nm), which is by far the most challenging region for all the optics of SITELLE and the telescope. A lower than expected transmission of the beamsplitter antireflection coating or the telescope mirrors might explain this difference. Note in particular that the reflectivity in the blue band of the telescope mirrors is significantly reduced a few years after the aluminizing process.

In any case, we can clearly see that the photon noise prevents a precise measurement of $\epsilon$. We thus have fitted the obtained data with a low order polynomial. Multiple correction functions have been obtained in the most used filters and they do not show any trend which is not described by a low order polynomial. The difference between the obtained functions in the same filters can thus be considered as a conservative measurement of the precision considering the fact that, in more than $4 \mathrm{yr}$, the primary mirror has been aluminized two times. The two blue filters ( $\mathrm{SN} 1$ and $\mathrm{C} 1$ ) display an uncertainty of up to 10 percent (which can in part be attributed to the aluminizing processes), but in the other filters the precision is better than 3 per cent.

Noise is very difficult to reduce when observing bright stars because of the detectors saturation. The only method that would seriously enhance the precision of the flux calibration function would be to observe at the same time a very high number of secondary calibration sources like star clusters for which standard spectra would have been obtained independently.

As the filter band is not the same everywhere in the field (see Fig. 12), the exact knowledge of $f_{\lambda}$ requires the determination of a 3D filter transmission function, which is still unknown. From Fig. 1, we can see that the edges of the filters are quite steep and the peak-to-peak difference in the band is not higher than 4 percent and generally smaller than 2 percent. We estimate that an error $\leq 5$ percent on the relative flux calibration may arise from this error.

\subsubsection{Absolute flux calibration}

The idea of the absolute flux calibration is to compute the mean modeling error function in the filter band by taking an image of a standard star that provides a direct measure of $N_{\mathrm{i} \text {, obs }}$.

From equation (12), we can see that this value can also be simulated. Considering $\epsilon(\sigma)=1$, we can calculate $N_{\mathrm{i} \text {, sim }}$

$N_{\mathrm{i}, \mathrm{sim}}=\int_{\sigma} \frac{f(\sigma)}{\sigma h c} S_{\text {prim. }}(\sigma) t(\sigma) G$.

From the ratio of the simulated value to the observed value of the instrumental flux of a standard star on the detectors we may estimate 

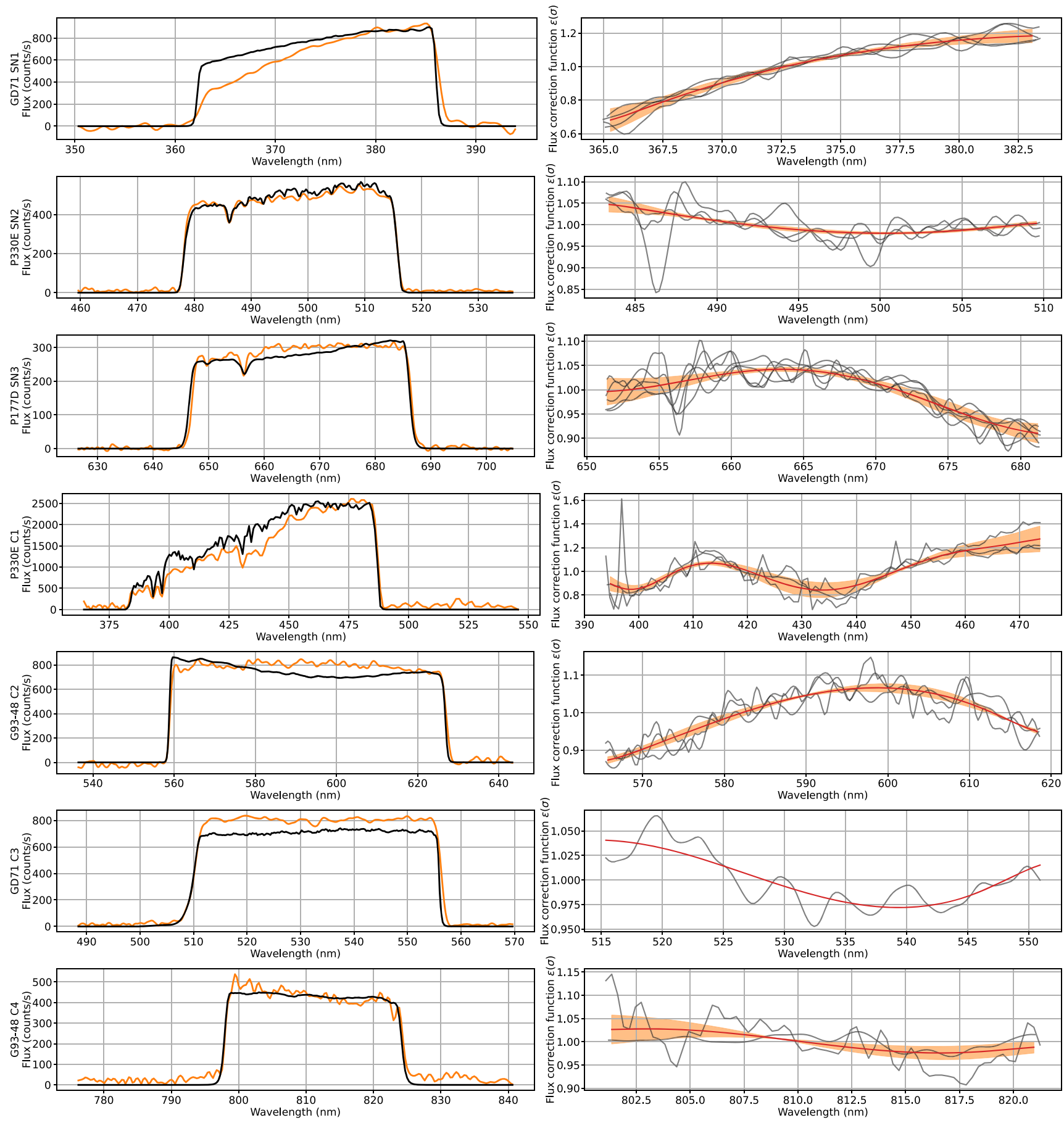

Figure 11. Flux correction functions computed for every SITELLE filter. Left: Observed (orange curve) and simulated (black curve) spectra of standard stars for each filter. The spectrophotometric standard spectra used for the simulation were obtained from different databases (Massey et al. 1988; Oke 1990; Bohlin, Gordon \& Tremblay 2014). The resolution of the observed spectra is $R=1000$ with the exception of SN3 and C4 $(R=3000)$ and C1 $(R=400)$. The resolution of the simulated spectra is reduced to the resolution of the observation. Right: Corresponding flux correction function $\epsilon(\sigma)$ computed from the ratio of both spectra (in black; see equation 17). Note that the obtained flux correction function has been divided by its mean to normalize the observed variations in the filter band. In all but one filters (the C3 filter) we obtained multiple correction functions, which are all represented as grey lines. All these flux correction functions were fitted with a polynomial (see text for details). One example of the fitted polynomials is in red. The orange region shows the maximum range of the flux calibration functions obtained.

the mean value of $\epsilon(\sigma)$ since, by definition,

$\overline{\epsilon(\sigma)}=\frac{N_{\mathrm{i}, \mathrm{obs}}}{N_{\mathrm{i}, \mathrm{sim}}}$
There are important limitations on the precision of the absolute flux calibration.

(i) Standard images are taken a few minutes after the end of the scan. However, in non-photometic conditions, atmospheric transmis- 


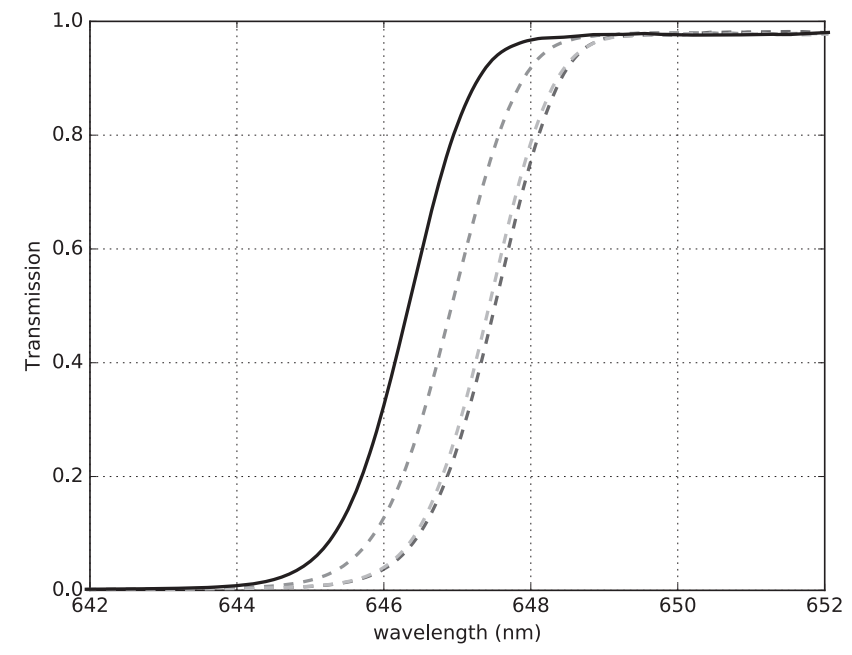

Figure 12. Edges of the SN3 filter at the centre (solid black) and different extreme positions in the field of view (dashed grey).

sion may change by 10 per cent on a minute time-scale in which case, the precision of the calibration cannot be guaranteed to be better than that.

(ii) The modulation efficiency is not measured at each scan, but the mean modulation efficiency is known to differ from as much as 5 per cent from one scan to another.

Given all these limitations, it is unclear whether or not this calibration step really enhances the precision of the calibration.

\subsection{Influence of the reduction steps on the flux calibration}

Flux calibration is certainly the most sensitive to the quality of each reduction step. We are going to analyse the relative contribution of each reduction step to its precision.

\subsubsection{Image correction and alignment}

As with any imager, the number of counts recorded in each pixel must first be corrected for electronic and thermal biases, coming from the detector, as well as differences in the illumination pattern due to the whole optical system comprising the telescope and the instrument optics. This corrected flux must also be conserved through the alignment steps that are all based on linear interpolation. All those first steps have an influence on the homogeneity of the measured flux in each image taken independently.

\subsubsection{Flat field}

With the possibly varying pixel-to-pixel modulation efficiency for high resolution data (see Section 4.2), flat-field correction is the other important contributor in terms of pixel-to-pixel flux calibration errors. Flat-field images are obtained by observing the sky at twilight through the same filter used to observe the science data. Five images are taken at the beginning of each night and combined to make a master flat-field image, which is used to correct the illumination pattern of the science images. The combination is an implementation of the averaged sigma-clipping algorithm of the task imcombine of the package STSDAS of the IRAF pipeline (Doug 1993). In the worst cases a gradient in the sky level of up to 5 per cent has be seen in the corrected images. The maximum difference is seen in the corners of the image.

\subsubsection{Alignment}

Once the alignment parameters are found by measuring the positions of the stars in the field of view with a Gaussian profile fitting algorithm, images are geometrically transformed with a classic linear interpolation algorithm. Even if linear interpolation certainly modifies the shape of a star and therefore cannot guarantee flux conservation, a simulation of this effect shows that the flux is perfectly well conserved for stars if aperture photometry is used, i.e. the total number of counts in a circular aperture around the star is conserved in an aperture larger than three times the FWHM. This result is obvious since the interpolation of any signal will conserve the value of the integral of the signal. ${ }^{9}$ We have therefore not detected any error on the flux measurement larger than the numerical error with aperture photometry. But, profile fitting will give a worse estimate of the flux since the overall shape is modified. For example, the peak value of the transformed point spread function (PSF) is around 0.5 the peak value of the real PSF. If a fitting procedure is used (in the idealized case of a Gaussian PSF), the median error made on the flux measurement is around -2 per cent (flux is always underestimated) and the largest error is always smaller than -5 percent. Note that we could enhance the quality of the profile fitting by using a model that would take the effect of the linear interpolation into account (i.e. the convolution of a 2D Gaussian PSF and a linear interpolation kernel).

\subsubsection{Cube combination}

During the combination step it is possible to correct for temporal variations of the atmospheric transmission. Remember that the cubes are complementary by definition: the light that does not go through one of the output ports must go through the other. Combining both cubes has therefore two advantages. (1) It provides twice as many photons, which enhances the SNR by a factor of $\sqrt{2}$. (2) With the assumption that the source is not variable, the variation of the sum of the flux measured on both ports provides a robust estimate of the variation of the mean sky transmission in the observed filter band (atmospheric extinction and airmass).

A first order combination equation of the two interferograms of the same source is

$I(t)=\frac{I_{1}(t)-I_{2}(t)}{I_{1}(t)+I_{2}(t)}$,

$I_{1}$ and $I_{2}$ being the interferograms recorded in camera 1 and camera 2, respectively (Davis et al. 2001; Martin et al. 2012); $t$ is the time when each sample is recorded. Because the interferograms measured on each arm are complementary (i.e. all the incoming flux is separated between each arm), $I_{1}-I_{2}$ represent the modulated part of the interferogram while $I_{1}+I_{2}$ is the intensity at the input of the interferometer. The relative variation of the atmospheric transparency is thus corrected by the denominator. As $I_{1}+I_{2}$ must be the same for all the pixels of the cube, we can use the stars to compute an atmospheric transmission function $T_{\text {atm. }}(t)=I_{1}(t)+I_{2}(t)$ for all the interferograms. We use the stars to compute the transmission because

${ }^{9}$ Linear interpolation kernels have unit response at zero frequency and hence conserve the integral over the full image, but power is spatially redistributed between the neighbouring pixels, so the aperture flux is conserved only if the limit where the flux lost through spatial redistribution at the border of the aperture is small compared to the flux integral within the aperture, which is the case in practice if the aperture is taken to be a few times the FWHM of the PSF. 

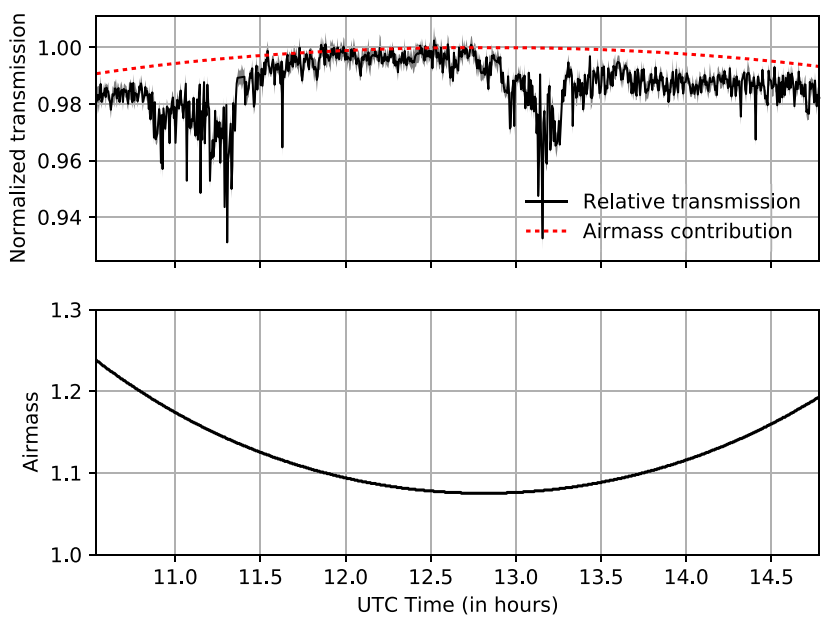

Figure 13. Top: Atmospheric transmission function during the acquisition of the M31 data cube in the SN3 filter observed on 2016 August 25 (Martin et al. 2018). The computed transmission is normalized to its 99th percentile. The grey surface represents the uncertainty. The airmass contribution to the atmospheric transmission is plotted in dotted red. It has been computed with a mean value of the extinction over Maunakea at the $\mathrm{H} \alpha$ wavelength of $6.2 \times 10^{-2} \mathrm{mag} \mathrm{am}^{-1}$ (Buton et al. 2012). Bottom: Airmass of the target. This figure is a reproduction of the fig. 5 of Drissen et al. (2019).

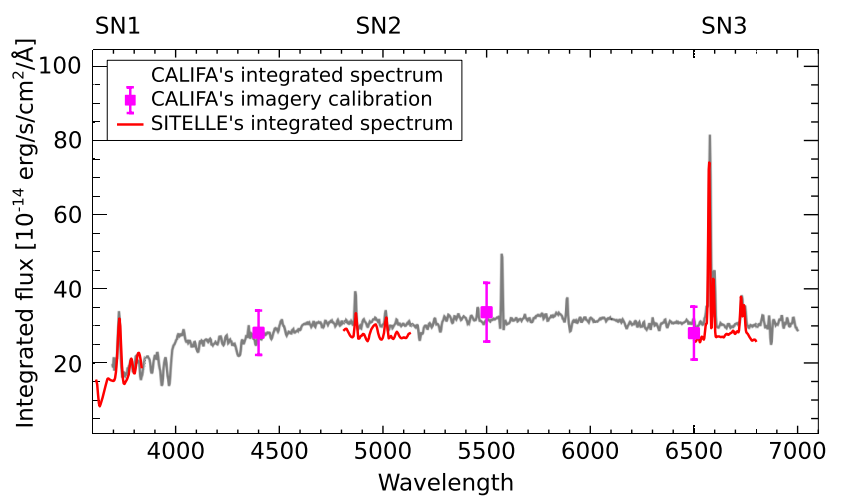

Figure 14. Integrated spectrum of NGC 628 obtained with SITELLE in three filters (SN1, SN2, and SN3) superimposed on the integrated spectra obtained with PPaK (Kelz et al. 2006; Sanchez et al. 2010). SITELLE's spectra have been convolved to respect PPaK's low resolution. A correction factor of 0.65 has been applied to consider PPaK's filling factor. The photometric calibration points used to calibrate PPAK spectrum are shown in purple along with their uncertainty. Part of the figure has been reproduced from Sanchez et al. (2010).

the measure of their flux is much less sensitive to scattered light. A typical atmospheric transmission function is shown in Fig. 13.

Since the reflectance $R$ and transmittance $T$ of the beamsplitter are not exactly equal to 50 percent, the energy transmitted in the unbalanced port is at most equal to $4 R T$ while the energy in the balanced port remains unaffected (see e.g. Bell 1972, p. 112). This difference in modulated energy and other possible differences in the transmission of the camera optics are taken into account by computing a modulation ratio $\left(\alpha=\overline{I_{2} / I_{1}}\right)$ by which the intensity on the unbalanced port is divided. Equation (20) can thus be rewritten

$I(t)=\frac{I_{1}(t)-I_{2}(t) / \alpha}{T_{\text {atm. }}(t)}$,

There is one underlying assumption in this equation: since the subtraction at the numerator will remove any amount of stray light
Table 3. Flux calibration checked against various references. In general, only individual or doublet line fluxes are compared. When the comparison covers the whole band the name of the filter is written instead.

\begin{tabular}{lcc}
\hline Object & Wavelength range & Error \\
\hline M33 PNe & $\mathrm{H} \alpha$ & $\mathbf{+ 2} \pm 7$ percent \\
& {$[\mathrm{O} \mathrm{IIII}] \lambda 5007$} & $\mathbf{+ 6} \pm 8$ per cent
\end{tabular}

Ciardullo et al. (2004)

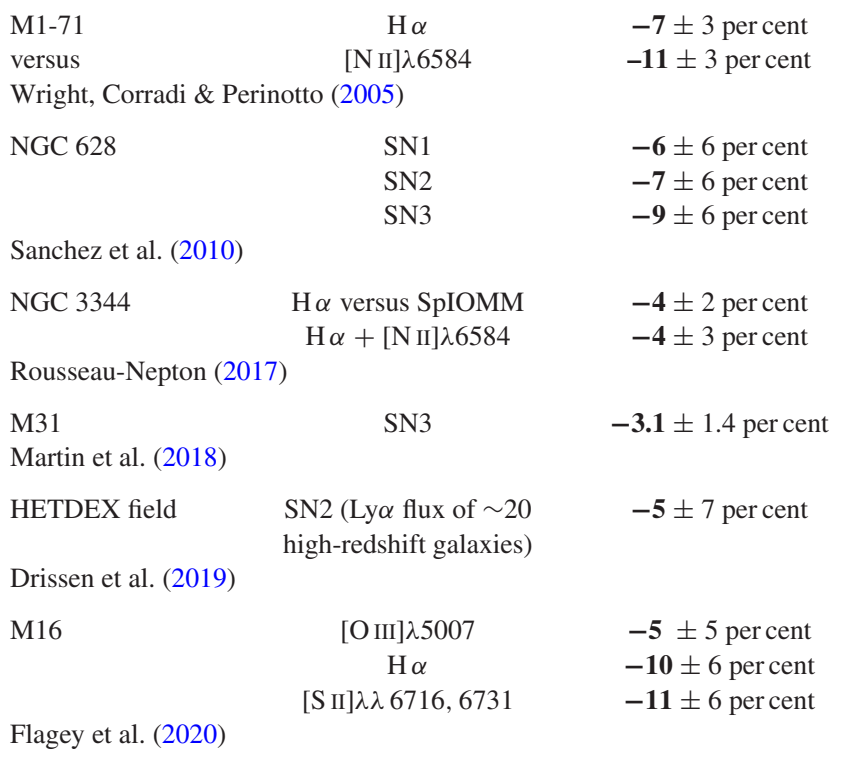

equally found in both cameras, the difference in the amount of stray light in both cameras must be negligible.

\subsubsection{Phase correction}

The error (in percentage) on the measure of the flux resulting from an error in the determination of the phase for each filter can be found in Table 1 . It is always smaller than 1 percent and can be safely neglected with respect to the other sources of uncertainty listed above.

\subsection{Assessment of the uncertainty}

\subsubsection{Uncertainty on the absolute flux calibration}

From the analysis conducted in this section we conclude that there are two major sources of uncertainty on the absolute flux calibration: the modulation efficiency measurement and the determination of the mean atmospheric transmission loss from standard star images. Potential phase correction errors ( $<1$ per cent) are completely negligible at this point.

We have checked the accuracy of the calibration against various references. All these results are reported in Table 3. There is an obvious general bias around -5 percent, which may come from an overestimation of the sky transparency and of the modulation efficiency. A better estimate of the modulation efficiency could be derived from the ratio of the total spectral energy present in the output spectra and the total energy deposited by the photons in the input interferograms. It could be taken into account in a future version of the reduction pipeline. We also must mention that a modulation efficiency loss of 10 percent has been measured during the observation of the SN3 cube of M31 (Martin et al. 2018). In 
general, the modulation efficiency does not vary much from one observation to another, which implies that, even if the estimate is biased, its precision is better than 5 per cent. However, a conservative evaluation of the uncertainty on the modulation efficiency should lead to a flux uncertainty between -10 percent and 0 percent. Laser frames are obtained at the beginning and the end of each scan, which should permit to calculate the modulation efficiency loss in future versions of the pipeline.

As for the measurement of the mean atmospheric transmission during the scan, we have also observed that, in rare cases, the standard star images were not taken right before or after the scan and sometimes a night later. In these cases, the measurement is unreliable and should not be trusted. The chosen method also suffers from the fact that the transmission can vary by more than 10 per cent on a time-scale of a few minutes, which means that the atmospheric transmission measured right after the scan sequence may not reflect precisely even the last minutes of the observation.

An evaluation of the precision of the flux calibration, taking the 5 per cent bias into account, is thus generally between -10 per cent and 0 percent if we consider the calibration checks reported in Table 3. But, a more conservative estimate should be considered to lie between -15 per cent and 5 percent.

In a general case, we recommend that the absolute flux calibration be checked against external data for now.

This uncertainty could certainly be reduced by providing an estimation of the mean modulation efficiency during each scan. In addition, preliminary results on the statistical relationship between the photometry of the stars present in the field of most science cubes in the SN3 and SN2 filters and the R, G, and I photometry of the Pan-STARRS catalogue (Chambers et al. 2016) indicates that the flux uncertainty in these two filters could be reduced to less than 5 per cent in most cases.

\section{WAVELENGTH CALIBRATION}

Recording an interferometric image implies to measure the flux at different angles $\theta$ with respect to the interferometer axis. At each step of the scan, the OPD $x_{\theta}$ with respect to the OPD $x$ measured on-axis is simply

$x_{\theta}=x \cos (\theta)$.

A demonstration of this formula is provided in Martin et al. (2018). If we consider a constant optical step size $\delta_{x},{ }^{10}$ at a given step index $j$, the on-axis OPD is

$x=j \delta_{x}$.

The maximum wavenumber $\sigma_{\max }$ that can be measured is inversely proportional to twice the sampling step size.

$\sigma_{\max , \theta}=\frac{1}{2 \delta_{x} \cos (\theta)}$

Like its predecessor, SpIOMM, SITELLE's observing mode makes use of spectral folding (Grandmont 2006; Drissen et al. 2010; Grandmont et al. 2012). Because the light is observed though a filter, one can discriminate between all the multiples of a given wavelength that do not fall into the observed band. It is therefore possible to scan

\footnotetext{
${ }^{10}$ In this paper only optical distances will be considered. The optical distance along the interferometer axis $x$ is roughly twice the mechanical position of the scanning mirror. Note also that all distances are always measured with respect to the $\mathrm{ZPD}$.
}

with a sampling step that is a multiple of the minimum observed wavelength and increase the resolution without folding the spectral information. The folding order $n$ of the observation is related to the number of times the step size is increased,

$\delta_{x, n}=(n+1) \delta_{x}$.

The maximum observable wavenumber, $\sigma_{\max , \theta}$, is then,

$\sigma_{\max , \theta}=\frac{n+1}{2 \delta_{x} \cos (\theta)}$.

If we want to make sure that all the wavelengths of the observed light are discriminated by the Fourier transform we must also limit the band to a minimum wavenumber, $\sigma_{\min , \theta}$, such that

$\sigma_{\min , \theta}=\frac{n}{2 \delta_{x} \cos (\theta)}$.

It follows that the wavenumber associated to a channel $i$ of an $N$ channels output spectrum is

$$
\begin{aligned}
\sigma_{i, \theta} & =\sigma_{\min , \theta}+\frac{i}{N}\left(\sigma_{\max , \theta}-\sigma_{\min , \theta}\right) \\
& =\frac{1}{2 \delta_{x} \cos (\theta)}\left(n+\frac{i}{N}\right) .
\end{aligned}
$$

We see that, to make an absolute calibration, the value of the incident angle of the light for each pixel of the cube is the only quantity needed. Inversely, if one measures the exact position, in channels, of the centroid of a line with a known wavelength, the incident angle of the spectrum can be derived from equation (29). From this equation we can directly relate the wavenumber, $\sigma$, of source measured at an angle $\theta$ with respect to the axis of the interferometer to its real wavenumber $\sigma_{0}$

$\sigma=\sigma_{0} \cos (\theta)$.

The zero-point must therefore be calibrated for each spectrum of the cube via the observation of a laser source at zenith. One source of uncertainty comes from the fact that the deformation of the optical structure when the telescope moves from the zenith position to the direction of the source has a strong impact on the incident angle seen by one pixel. By comparing calibration maps obtained at 47 degrees in four directions (north, south, east, and west) we have found that this calibration method results in a gradient error in the relative wavelength calibration no higher than $25 \mathrm{~km} \mathrm{~s}^{-1}$. This case is well illustrated by fig. 6 of Flagey et al. (2020) who compare the effect of a wavelength calibration based on a laser cube obtained at zenith and a laser cube obtained with the telescope pointing in the same direction as the science target. The error gradient is clearly visible, but is no higher than a few $\mathrm{km} \mathrm{s}^{-1}$.

Another source of absolute calibration uncertainty is the lack of precision on the calibration laser wavelength. The error on the velocity measurement $\epsilon_{v}$ is related to the error on the calibration laser wavenumber, $\epsilon_{\sigma}$, since

$\epsilon_{v}=c \frac{\epsilon_{\sigma}}{\sigma_{\text {laser }}}$,

with $\sigma_{\text {laser }}$ the real wavenumber of the calibration laser. Therefore, an error of $0.1 \mathrm{~nm}$ on the calibration laser wavelength translates into an error of $55 \mathrm{~km} \mathrm{~s}^{-1}$. This bias can be corrected by measuring the velocity of the Meinel $\mathrm{OH}$ bands in a few SN3 cubes. The reduction pipeline uses the manufacturer value of $543.5 \mathrm{~nm}$, which appears to be biased by $80 \pm 10 \mathrm{~km} \mathrm{~s}^{-1}$.

The last source of calibration uncertainty comes from the phase correction, which may induce a shift in the measured wavelength of up to 10 percent of the FWHM, which means e.g. $6 \mathrm{~km} \mathrm{~s}^{-1}$ at 


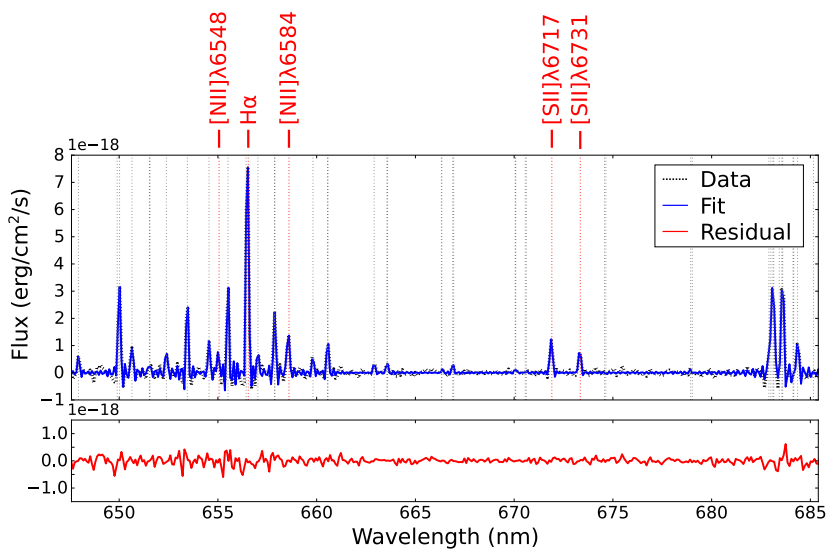

Figure 15. Example of a fit of the Meinel $\mathrm{OH}$ bands of a sky spectrum in the field of IC 348. $R=4500$ (courtesy of Gregory Herczeg). The fitted emission lines of the diffuse gas around the nebula are shown.

a resolution of 5000. Note that, however, most velocity studies are based on the $\mathrm{H} \alpha$ line, which is found in the SN3 filter and suffers a maximum deviation of 5 per cent of the FWHM, i.e. $3 \mathrm{~km} \mathrm{~s}^{-1}$ at a resolution of 5000 .

We have compared the velocity of 124 planetary nebulæ (PNe) detected with SITELLE in the centre of M31, from a low resolution data cube obtained during the commissioning, with the velocity measured by Merrett et al. (2006). 86 of the 124 PNe show a compatible velocity within the uncertainties (see Fig. 16). Note that the bias induced by the manufacturer value of the calibration laser wavelength was corrected, but no other correction was made. However, this basic wavelength calibration can be further improved to a precision of a few $\mathrm{km} \mathrm{s}^{-1}$ by fitting the Meinel $\mathrm{OH}$ bands that are generally present everywhere in the cube (especially in the SN3 red filter, see Fig. 15). A calibration laser map model has been developed to increase the precision of the calibration and reconstruct the velocity field in regions where the $\mathrm{OH}$ lines are not visible (Martin et al. 2018). This operation can be easily done with ORCS (Martin et al. 2015) and has been used by e.g. Martin et al. (2016, 2018), Shara et al. (2016), Rousseau-Nepton et al. (2017), Gendron-Marsolais et al. (2018), and Duarte Puertas et al. $(2019,2021)$.

\section{ASTROMETRIC CALIBRATION}

Astrometric calibration is computed from the fit of the point-like sources detected in the field of view and the transformation of their celestial coordinates (Greisen \& Calabretta 2002) found in the GaiaDR2 catalogue (Brown et al. 2018). The fitting engine fits all the stars at the same time, which enhances the precision of the transformation parameters. The precision of the astrometric calibration is limited to 3 pixels $(\sim 1 \operatorname{arcsec})$ in an 11 arcmin circle around the centre of the field by the optical distortions, which are not taken into account in the reduction pipeline (i.e. only a linear astrometric solution is computed, which leaves residual errors especially at the edge of the field of view; see Fig. 17). Tools are distributed with ORCS (Martin et al. 2015) to compute a distortion model and optimize the astrometric calibration (see Martin et al. 2018).

\section{CONCLUSIONS}

We have discussed in detail the quality of SITELLE calibration as obtained with the reduction pipeline. All the known sources of uncertainty during the reduction process have been explained and

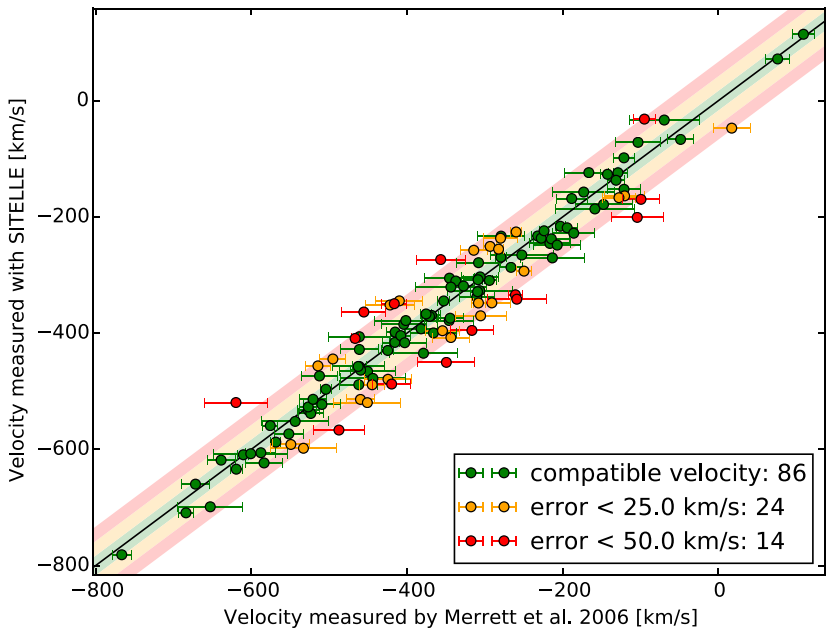

Figure 16. Comparison of the measured velocity of 124 planetary nebulae detected with SITELLE in M31 with the measurement of Merrett et al. (2006). The resolution of the cube is 400 . The one-to-one line is indicated by a black line.

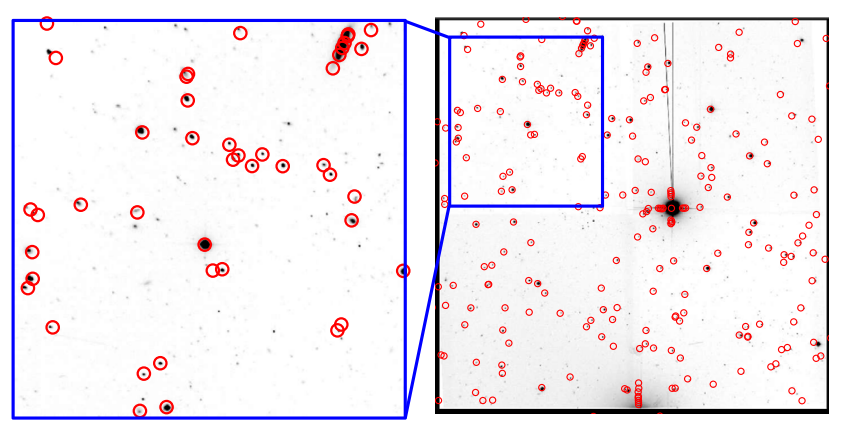

Figure 17. Positions of the stars from the Gaia-DR2 catalogue transformed with the computed world coordinate system of the field around the planetary nebula M1-71.

quantified. We have also described the most important steps of the reduction pipeline ORBS and provided the core concepts behind the papers describing refined algorithms developed to enhance the wavelength and astrometric calibrations (Martin et al. 2018) and optimize the extraction of emission line parameters (Martin et al. 2016).

We have shown that the absolute flux calibration uncertainty was to be considered between -15 per cent and 5 percent. The general bias is likely to be corrected in the next versions via a more precise evaluation of the modulation efficiency. We thus recommend that the flux calibration be checked against external data. An error gradient on the basic wavelength calibration has been observed to be as large as $15 \mathrm{~km} \mathrm{~s}^{-1}$, but it can be corrected by measuring the velocity of Meinel $\mathrm{OH}$ bands in the cube (especially in the SN3 filter). This operation can be done with the program built for the analysis of SITELLE data ORCS (Martin et al. 2018). The astrometric calibration was done via the comparison with the Gaia-DR2 catalogue and is limited to a precision of $\sim 1$ arcsec by the optical distortions, which are not corrected by the reduction pipeline. Tools are provided with ORCS to compute a distortion model and enhance the astrometric calibration (Martin et al. 2018). 


\section{ACKNOWLEDGEMENTS}

This paper is based on observations obtained with SITELLE, a joint project of Université Laval, ABB, Université de Montréal, and the Canada-France-Hawaii Telescope (CFHT) which is operated by the National Research Council (NRC) of Canada, the Institut National des Science de l'Univers of the Centre National de la Recherche Scientifique (CNRS) of France, and the University of Hawaii. The authors wish to recognize and acknowledge the very significant cultural role that the summit of Maunakea has always had within the indigenous Hawaiian community. LD is grateful to the Natural Sciences and Engineering Research Council of Canada, the Fonds de Recherche du Québec, and the Canadian Foundation for Innovation for funding.

\section{DATA AVAILABILITY}

The data underlying this article will be shared on reasonable request to the corresponding author.

\section{REFERENCES}

Baril M. R. et al., 2016, in Evans C. J., Simard L., Takami H., eds, Ground-based and Airborne Instrumentation for Astronomy VI. SPIE, Bellingham, p. 990829

Bell R. J., 1972, Introductory Fourier Transform Spectroscopy. Academic Press, New York, USA

Bohlin R. C., Gordon K. D., Tremblay P. E., 2014, PASP, 126, 711

Brault J., 1987, Mikrochimica Acta, 3,1

Brown A. G. A. et al., 2018, A\&A, 616, A1

Buton C. et al., 2012, A\&A, 549, A8

Chambers K. C. et al., 2016, preprint (arXiv:1612.05560)

Chase D. B., 1982, Appl. Spectrosc., 36, 240

Ciardullo R., Durrell P. R., Laychak M. B., Herrmann K. A., Moody K., Jacoby G. H., Feldmeier J. J., 2004, ApJ, 614, 167

Cooley J., Tukey J. W., 1965, Math. Comput., 19, 297

Davis S. P., Abrams M. C., Brault J. W. J. W., 2001, Fourier Transform Spectrometry. Academic Press, San Diego

Doug T., 1993, in Hanisch R. J., Brissenden R. J. V., Barnes J., eds, ASP Conf. Ser. Vol. 52, Astronomical Data Analysis Software and Systems II. Astron. Soc. Pac., San Francisco, p. 173

Drissen L., Bernier A.-P., Rousseau-Nepton L., Alarie A., Robert C., Joncas G., Thibault S., Grandmont F., 2010, in McLean I. S., Ramsay S. K., Takami H., eds, Proc. SPIE Conf. Ser. Vol. 7735, Astronomical Telescopes + Instrumentation. SPIE, Bellingham, p. 77350B

Drissen L. et al., 2019, MNRAS, 485, 3930

Duarte Puertas S., Iglesias-Páramo J., Vilchez J. M., Drissen L., Kehrig C., Martin T., 2019, A\&A, 629, A102

Duarte Puertas S., Vilchez J. M., Iglesias-Páramo J., Drissen L., Kehrig C., Martin T., Pérez-Montero E., Arroyo-Polonio A., 2021, A\&A, 645, A57

Flagey N., McLeod A. F., Aguilar L., Prunet S., 2020, A\&A, 635, A111

Forman M. L., Steel W. H., Vanasse G. A., 1966, J. Opt. Soc. Am., 56, 59

Fulton T. et al., 2016, MNRAS, 458, 1977

Gendron-Marsolais M. et al., 2018, MNRAS, 479, L28

Grandmont F., 2006, PhD thesis, Université Laval

Grandmont F., Drissen L., Mandar J., Thibault S., Baril M. R., 2012, in McLean I. S., Ramsay S. K., Takami H., eds, Proc. SPIE Conf. Ser. Vol. 8446, Ground-based and Airborne Instrumentation for Astronomy IV. SPIE, Bellingham, p. $84460 \mathrm{U}$

Greisen E. W., Calabretta M. R., 2002, A\&A, 395, 1061
Kelz A. et al., 2006, Publ. Astron. Soc. Pac., 118, 129

Learner R. C. M., Thorne A. P., Wynne-Jones I., Brault J. W., Abrams M. C., 1995, J. Opt. Soc. Am., 12, 2165

Mandar J., 2012, PhD thesis, Université Laval

Martin T., 2015, Phd thesis, Université Laval

Martin T., Drissen L., 2016, in Reylé C., Richard J., Cambrésy L., Deleuil M., Pécontal E., Tresse L., Vauglin I., eds, Proceedings of the Annual Meeting of the French Society of Astronomy \& Astrophysics. French Society of Astronomy \& Astrophysics, Paris, p. 23

Martin T., Drissen L., Joncas G., 2012, in Radziwill N. M., Chiozzi G., eds, Proc. SPIE Conf. Ser. Vol. 2, Software and Cyberinfrastructure for Astronomy II. SPIE, Bellingham, p. 84513K

Martin T., Drissen L., Joncas G., 2015, Astronomical Data Analysis Software an Systems XXIV (ADASS XXIV). Astronomical Society of the Pacific, San Francisco, California, p. 495

Martin T. B., Prunet S., Drissen L., 2016, MNRAS, 463, 4223

Martin T. B., Drissen L., Melchior A.-L., 2018, MNRAS, 473, 4130

Martin T., Milisavljevic D., Drissen L., 2021, MNRAS, 502, 1864

Massey P., Strobel K., Barnes J. V., Anderson E., 1988, ApJ, 328, 315

Merrett H. R. et al., 2006, MNRAS, 369, 120

Mertz L., 1967, Infrared Phys., 7, 17

Michaelian K. H., 1989, Infrared Phys., 29, 87

Oke J. B., 1990, AJ, 99, 1621

Rousseau-Nepton L., 2017, Phd thesis, Université Laval

Rousseau-Nepton L., Robert C., Drissen L., Martin R. P., Martin T., 2017, MNRAS, 477, 4152

Sakai H., Vanasse G. A., Forman M. L., 1968, J. Opt. Soc. Am., 58, 84

Sanchez S. F., Rosales-Ortega F. F., Kennicutt R. C., Johnson B. D., Diaz A. I., Pasquali A., Hao C. N., 2010, MNRAS, 410, 313

Shara M. M., Drissen L., Martin T., Alarie A., Stephenson F. R., 2016, MNRAS, 465, 739

Wright S. a., Corradi R. L. M., Perinotto M., 2005, A\&A, 436, 9

\section{APPENDIX: EFFECTS OF A PHASE ERROR ON THE ILS}

The effect of an error on $\phi_{0}$ can be studied by considering an erroneous phase $\phi(\sigma)=\delta p_{0}$ applied to a perfect interferogram. Equation (4) can then be written

$$
\begin{aligned}
S(\sigma) & =\operatorname{Re}\left(\hat{I}(\sigma) e^{-i \delta p_{0}}\right) \\
& =\hat{I}_{\operatorname{Re}}(\sigma) \cos \left(\delta p_{0}\right)+\hat{I}_{\mathrm{Im}}(\sigma) \sin \left(\delta p_{0}\right) .
\end{aligned}
$$

This will result in mixing the real and imaginary parts of the spectrum (see Fig. A1). The error made on the line amplitude is then

$\Delta_{\text {Flux }}[$ per cent $]=100 \times\left[1-\cos \left(\frac{\delta p_{0}}{2}\right)\right]$.

The error made on the line centroid is, in percentage of the FWHM,

$\Delta_{\text {Centroid }}[$ per cent $]=39.1 \times \delta p_{0}$.

These relations have been empirically calculated from a numerical simulation. The results of the numerical simulation are plotted in Fig. A1. As the phase is a slowly varying function of the wavelength, these relations can be used to compute the effect of a phase error no larger than $\pi / 4$ at a given wavelength (the phase error is then considered as locally constant). As one can see on Fig. A1, when the phase error is larger than $\pi / 4$ the negative lobe becomes important and even the notion of 'line' starts to be questionable. 

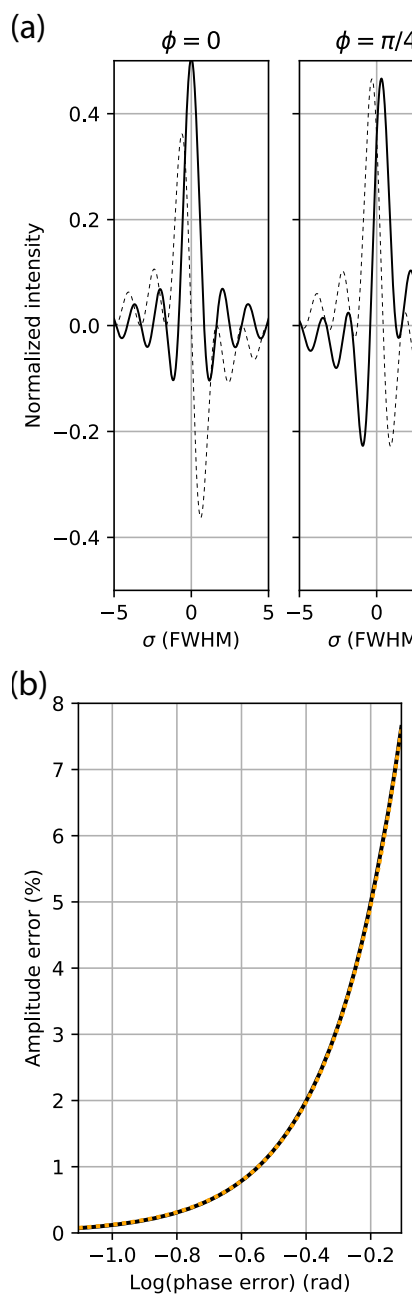
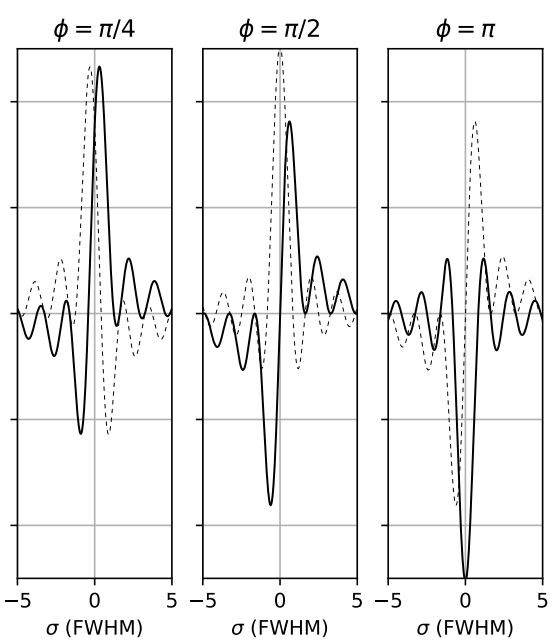

(c)

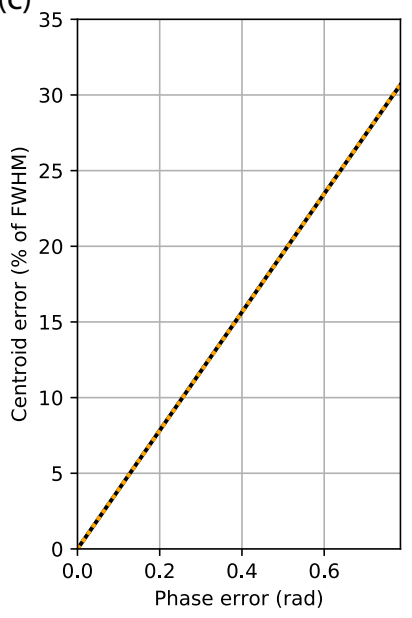

Figure A1. (a) Effect of a constant phase error $\Phi(\sigma)$ on the ILS. The real part is drawn in solid black and the imaginary part is dotted. The wavenumber $\sigma$ is given in units of the line's FWHM. (b) Effect of a constant phase error on the amplitude of the line. The black line represents the numerical simulation and the dotted orange line represents the model computed with equations (A3) and (A4) (the numerical simulation and the model are perfectly superposed). The phase error (log scale) goes from $0.1 \pi / 4$ to $\pi / 4$. (c) Effect of a constant phase error on the measured position in percentage of the line FWHM.

This paper has been typeset from a $\mathrm{T}_{\mathrm{E}} \mathrm{X} / \mathrm{LT}_{\mathrm{E}} \mathrm{X}$ file prepared by the author. 